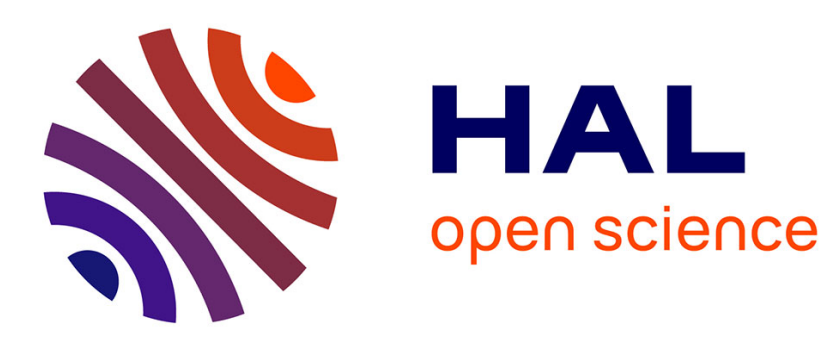

\title{
Influence de l'orientation dendritique sur la cinétique d'oxydation à haute température d'alliages de nickel contenant des carbures
}

Patrice Berthod, Sébastien Noël, Lionel Aranda

\section{To cite this version:}

Patrice Berthod, Sébastien Noël, Lionel Aranda. Influence de l'orientation dendritique sur la cinétique d'oxydation à haute température d'alliages de nickel contenant des carbures. Annales de Chimie Science des Matériaux, 2008, 33 (1), pp.59 - 80. 10.3166/acsm.33.59-80 . hal-02164329

\section{HAL Id: hal-02164329 \\ https://hal.science/hal-02164329}

Submitted on 25 Jun 2019

HAL is a multi-disciplinary open access archive for the deposit and dissemination of scientific research documents, whether they are published or not. The documents may come from teaching and research institutions in France or abroad, or from public or private research centers.
L'archive ouverte pluridisciplinaire HAL, est destinée au dépôt et à la diffusion de documents scientifiques de niveau recherche, publiés ou non, émanant des établissements d'enseignement et de recherche français ou étrangers, des laboratoires publics ou privés. 


\title{
INFLUENCE DE L'ORIENTATION DENDRITIQUE SUR LA CINETIQUE D'OXYDATION A HAUTE TEMPERATURE D'ALLIAGES DE NICKEL CONTENANT DES CARBURES
}

\author{
Patrice BERTHOD, Sébastien NOEL, Lionel ARANDA
}

Laboratoire de Chimie du Solide Minéral (U.M.R. 7555), Faculté des Sciences et Techniques, Université Henri Poincaré Nancy 1, BP 239, 54506 Vandoeuvre-Lès-Nancy Cedex, France.

\begin{abstract}
Influence of the dendrites orientation on the high temperature oxidation kinetic of ternary nickel-base alloys containing chromium carbides - Two chromium-containing nickel alloys with two carbides densities, were elaborated by casting. Two types of sample for thermogravimetry tests were cut following two orientations with regard to the external surface, in order to allow the uncoupling of constants. Oxidation tests were performed at 1000, 1100 and $1200^{\circ} \mathrm{C}$ for 50 hours and both transient oxidation and parabolic oxidation constants were studied. For the alloy with the lowest carbon content, the oxidation rate of the sample for which the main surface is parallel to the underlying dendrites is higher than for the other sample with a main surface perpendicular. On the contrary, oxidation of the alloy with the highest carbon content is faster when the external surface is mainly perpendicular to dendrites. These two observations are enhanced for the uncoupled constants. For both alloys, the oxidation rate seems to be governed by two levels of activation energy related to the two orientations. A perpendicular orientation and a more dense carbides network both lead to higher diffusion coefficients for chromium in the sub-surface.
\end{abstract}

Résumé - Deux alliages de nickel avec la même teneur en chrome et avec deux densités de carbures ont été élaborés par fonderie et testés en oxydation à haute température. Les essais thermogravimétriques ont été réalisés à 1000,1100 et $1200^{\circ} \mathrm{C}$, sur deux types d'échantillons. Ces derniers ont été découpés dans les lingots pour que la surface extérieure soit majoritairement parallèle ou perpendiculaire aux dendrites. Les constantes d'oxydation obtenues ont pu être découplées pour préciser la contribution de chaque type de surface. Pour l'alliage à faible taux de carbures la cinétique d'oxydation est plus grande pour une surface majoritairement parallèle aux dendrites tandis que c'est le contraire pour l'alliage à fort taux de carbures. Il en est de même pour les constantes découplées. Pour chaque alliage, l'énergie d'activation semble être liée à l'orientation dendritique. La diffusion du chrome au travers de la zone affectée par l'oxydation est plus rapide quand l'alliage contient plus de carbures et quand la surface est perpendiculaire aux dendrites.

Tirés à part : Patrice BERTHOD, LCSM, B.P. 239 - 54506 Vandoeuvre-lès-Nancy, France 


\section{INTRODUCTION}

La microstructure des alliages métalliques obtenus par fonderie classique dépend de leur composition chimique pour ce qui est de la nature des phases présentes. Par contre sa texture en un lieu particulier résulte plus des conditions de solidification locales, notamment du gradient thermique local. Ce dernier, en effet, impose une direction générale à la croissance cristalline, et donc aux dendrites et aux joints de grains [1-3], un effet illustré par la figure 1. Il est connu que les joints de grains jouent un grand rôle dans la diffusion des espèces impliquées dans les phénomènes d'oxydation [4,5]. De ce fait l'orientation de ces chemins diffusionnels privilégiés est susceptible d'intervenir au niveau de la cinétique locale de l'oxydation à haute température des superalliages de fonderie. Cette dernière peut ainsi dépendre de l'orientation dendritique par rapport à la partie de surface considérée, comme cela avait été déjà observé sur des superalliages de compositions complexes [6].

Afin d'étudier cet effet possible dans un contexte plus favorable à l'analyse, par le choix d'une composition chimique simplifiée permettant une étude facilitée du comportement du chrome, deux alliages ternaires ont été élaborés par fonderie et des échantillons préparés puis testés en oxydation à haute température dans des conditions propres à mettre en relief le comportement de surfaces orientées de façon précise par rapport aux dendrites sous-jacentes. Ces alliages simplifiés ont été choisis de façon à présenter un caractère formeur de chromine et à le conserver jusqu'à la fin des essais d'oxydation : à base de nickel (de préférence au cobalt ou au fer par exemple), avec 30\% de chrome, et deux teneurs en carbone pouvant être considérées comme les bornes de l'intervalle des teneurs de la majorité des superalliages industriels renforcés par carbures [7-8].
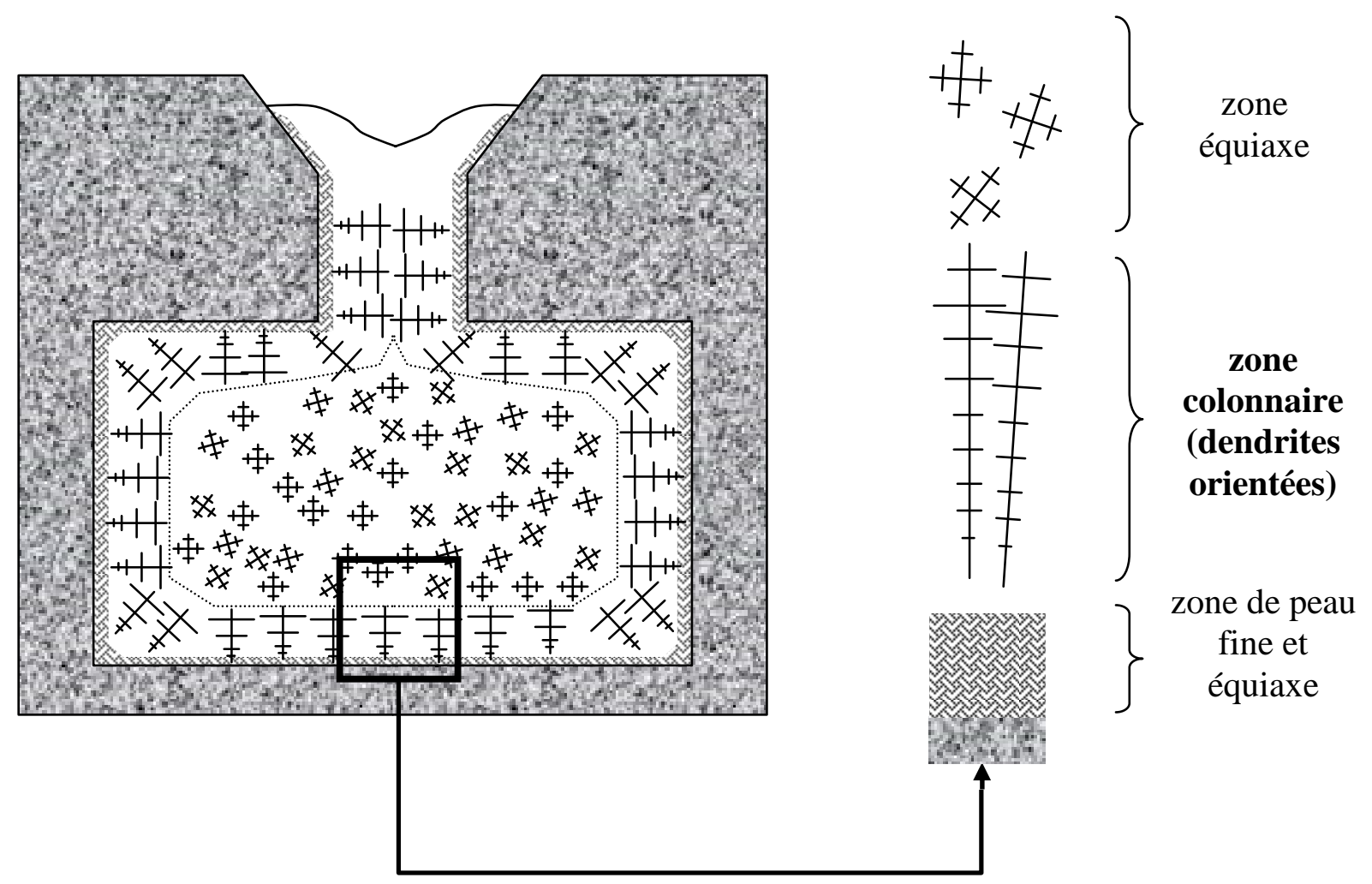

Figure 1. Evolution depuis le moule de la texture microstructurale dans l'alliage de fonderie 


\section{DETAILS EXPERIMENTAUX}

\subsection{Elaboration des alliages et préparation des échantillons pour essais thermogravimétriques}

Deux alliages ont été élaborés, l'un contenant $0,2 \%$ massiques de carbone et l'autre en contenant $0,8 \%$. Tous deux sont à base de nickel (environ $70 \%$ massiques) et contiennent $30 \%$ massiques de chrome. Fusion et solidification ont été réalisées dans le creuset froid d'un four à induction à haute fréquence CELES, sous une atmosphère inerte d'environ 300 millibars d'argon U (Ar, $\mathrm{H}_{2} \mathrm{O}<3 \mathrm{ppm}, \mathrm{O}_{2}<2 \mathrm{ppm}, \mathrm{C}_{\mathrm{n}} \mathrm{H}_{\mathrm{m}}<0,5 \mathrm{ppm}$ ). La fusion des charges (100g au total) initialement constituées de nickel (Eramet, > 99,9\%), de chrome (Alfa Aesar, > 99,9\%) et de carbone (graphite) a conduit à l'obtention de lingots de forme compacte et de dimensions suffisantes pour en extraire les échantillons pour les essais d'oxydation.

Les échantillons destinés aux essais thermogravimétriques, parallélépipèdes de dimensions approximatives $10 \times 10 \times 2 \mathrm{~mm}^{3}$ ont été découpés de deux manières différentes de façon à obtenir une surface extérieure majoritairement parallèle ou majoritairement perpendiculaire aux dendrites sous-jacentes (figure 2). Avant essai d'oxydation chaque échantillon a été poli jusqu'au papier SiC de grade 1200, avec émoussement des arêtes et coins.

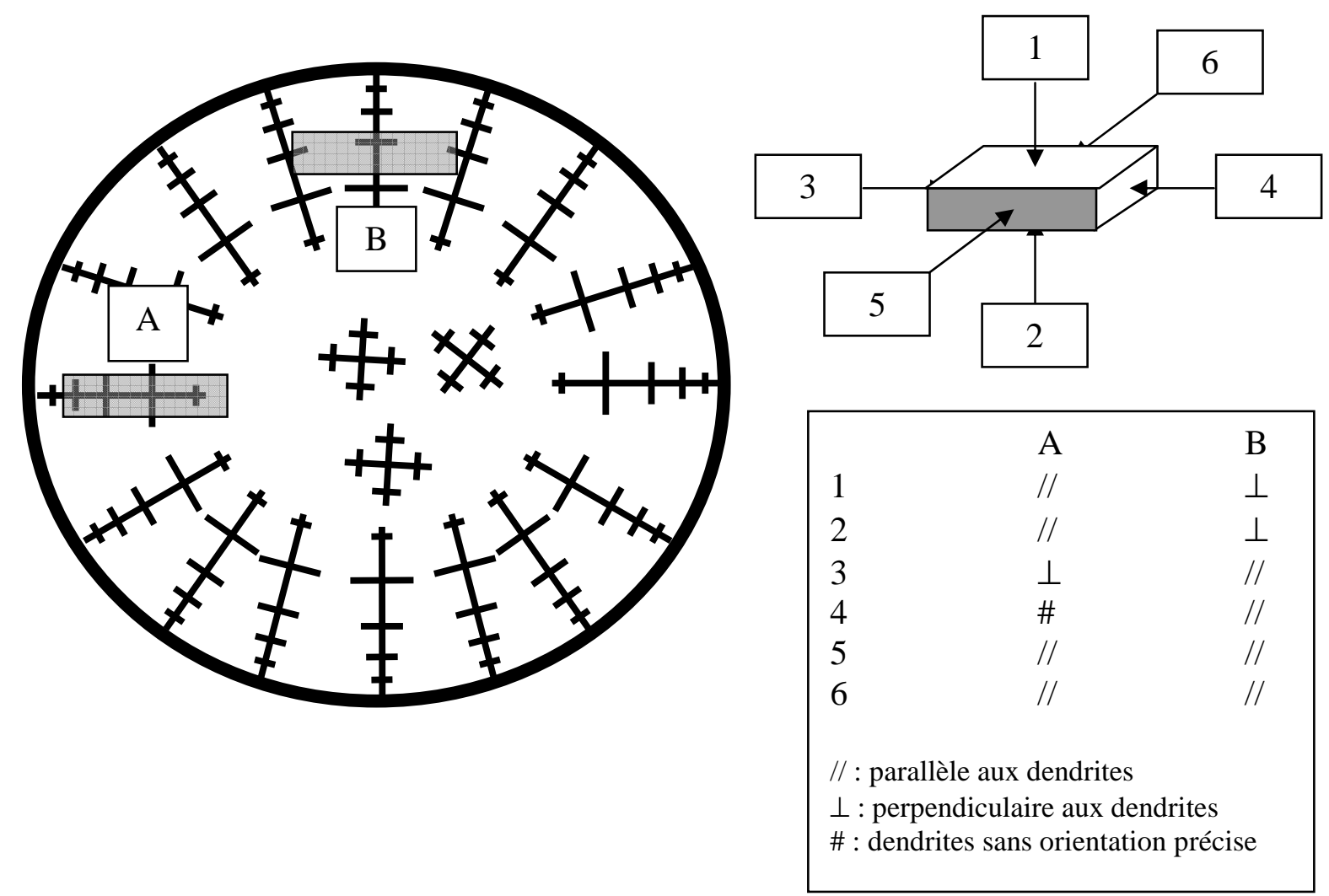

Figure 2. Mode de découpe des deux types d'échantillons pour essais thermogravimétriques

\subsection{Réalisation des essais thermogravimétriques}

Les essais thermogravimétriques ont été réalisés sur chaque alliage à 1000,1100 et $1200^{\circ} \mathrm{C}$ pour les deux types d'orientation d'échantillon. La thermobalance utilisée était une Setaram TGA 
92, et l'oxydation avait lieu dans une atmosphère d'air synthétique $\left(80 \% \mathrm{~N}_{2}-20 \% \mathrm{O}_{2}\right)$ circulant avec un débit de 1,5 litres/heure. Dans tous les cas la durée d'essai a été de 50 heures, durée assez longue pour permettre l'obtention d'un régime de prise de masse parabolique sur une durée suffisante et restant courte de façon à ne pas rencontrer de problèmes de décollement local de la couche d'oxyde susceptible de détériorer la courbe et de conduire à une déchromisation hétérogène de la surface.

Avant d'être traitées, les mesures thermogravimétriques brutes ont été corrigées des pertes de masse accompagnant les phénomènes de volatilisation de la suspension de platine et de celle de la chromine (réoxydation de $\mathrm{Cr}_{2} \mathrm{O}_{3}$ en $\mathrm{CrO}_{3}$ gazeux au-delà de $1000^{\circ} \mathrm{C}$ ). Des essais «à blanc » (suspension seule, sans échantillon) ont conduit aux valeurs suivantes de vitesse de volatilisation de la suspension: $\mathrm{K}_{\mathrm{Pt}}(\mathrm{t})=0,93 \cdot 10^{-10}, 4,5 \cdot 10^{-10}$ et $26,3 \cdot 10^{-10} \mathrm{~g} \cdot \mathrm{s}^{-1}$ pour 1000,1100 et $1200^{\circ} \mathrm{C}$ respectivement, qui ont été utilisées pour additionner un terme $\mathrm{K}_{\mathrm{Pt} . \mathrm{t}}$ aux mesures de masse avant division par la surface d'échantillon. Pour la perte de masse par volatilisation de la chromine, ce sont des valeurs mesurées antérieurement sur des alliages similaires qui ont été utilisées. Pour l'alliage à $0,2 \% \mathrm{C}$ un terme $\mathrm{Kv} . \mathrm{t}$ avec $\mathrm{Kv}=20.10^{-10}, 63 \cdot 10^{-10}$ et $149 \cdot 10^{-10} \mathrm{~g} \cdot \mathrm{cm}^{-2} \cdot \mathrm{s}^{-1}$ pour 1000,1100 et $1200^{\circ} \mathrm{C}$ a été additionné aux mesures de masse après division par la surface de l'échantillon. Pour l'alliage à $0,8 \% \mathrm{C}$ les valeurs de $\mathrm{Kv}$ utilisées ont été de $16 \cdot 10^{-10}, 91 \cdot 10^{-10}$ et $165 \cdot 10^{-10} \mathrm{~g} \cdot \mathrm{cm}^{-2} \cdot \mathrm{s}^{-1}$.

A partir du début du palier en température, et après éventuellement l'achèvement d'un régime linéaire transitoire, toute la surface de l'échantillon suit un régime parabolique de prise de masse par oxydation suivant la loi parabolique corrigée de la perte de masse par volatilisation de la chromine (1), $\Delta \mathrm{m} / \mathrm{S}(\mathrm{t})$ désignant la prise de masse par unité de surface due à l'oxydation, et le terme -Kv.t représentant la perte de masse induite par la volatilisation de la chromine. Cette prise de masse est en fait la somme de deux contributions qui peuvent être différentes, $\Delta \mathrm{m}_{/ /} / \mathrm{S}_{/ /}(\mathrm{t})$ et $\Delta \mathrm{m}_{\perp} / \mathrm{S}_{\perp}(\mathrm{t})$. Ces derniers décrivent les prises de masse spécifiques de la surface parallèle aux dendrites qui lui sont sous-jacentes (2) et de la surface perpendiculaire (3) respectivement, d'où l'équation (4). Les égalités (1) et (4) conduisent à l'équation (5) dans laquelle le terme de volatilisation disparaît et qui peut être écrite deux fois pour un même couple (alliage, température) $\mathrm{du}$ fait qu'il y a deux types d'échantillon. Les deux équations linéaires ainsi obtenues sont indépendantes (rapports <surface parallèle sur surface perpendiculaire> différents pour les deux types d'échantillon) et la résolution du système conduit aux valeurs des constantes paraboliques spécifiques de la surface de type parallèle aux dendrites $\left(\mathrm{Kp}_{/ /}\right)$et de la surface de type perpendiculaire aux dendrites $\left(\mathrm{Kp}_{\perp}\right)$. Les valeurs obtenues pour toutes ces constantes paraboliques seront affectées d'une incertitude de $\pm 13 \%$, écart-type qui avait été antérieurement précisé au moyen d'analyses thermogravimétriques répétées réalisées à $1050^{\circ} \mathrm{C}$ avec la même thermobalance sur un même alliage appartenant à la même famille que ceux étudiés ici [9].

$$
\begin{aligned}
& \frac{\Delta \mathrm{m}}{\mathrm{S}}(\mathrm{t})=\sqrt{2 \cdot \mathrm{Kp}} \cdot \mathrm{t}^{\frac{1}{2}}-\mathrm{Kv} \cdot \mathrm{t} \quad \text { avec } \mathrm{S}=\mathrm{S}_{/ /}+\mathrm{S}_{\perp} \\
& \frac{\Delta \mathrm{m}_{/ /}}{\mathrm{S}_{/ /}}(\mathrm{t})=\sqrt{2 \cdot \mathrm{Kp}_{/ /}} \cdot \mathrm{t}^{\frac{1}{2}}-\mathrm{Kv} \cdot \mathrm{t} \quad \text { (2) } \quad \frac{\Delta \mathrm{m}_{\perp}}{\mathrm{S}_{\perp}}(\mathrm{t})=\sqrt{2 \cdot \mathrm{Kp}_{\perp}} \cdot \mathrm{t}^{\frac{1}{2}}-\mathrm{Kv} \cdot \mathrm{t} \\
& \frac{\Delta \mathrm{m}}{\mathrm{S}}(\mathrm{t})=\frac{1}{\mathrm{~S}} \cdot\left(\mathrm{S}_{/ /} \cdot \frac{\Delta \mathrm{m}_{/ /}}{\mathrm{S}_{/ /}}(\mathrm{t})+\mathrm{S}_{\perp} \cdot \frac{\Delta \mathrm{m}_{\perp}}{\mathrm{S}_{\perp}}(\mathrm{t})\right)=\frac{\mathrm{S}_{/ /}}{\mathrm{S}} \sqrt{2 \cdot \mathrm{Kp}_{/ /}} \cdot \sqrt{\mathrm{t}}+\frac{\mathrm{S}_{\perp}}{\mathrm{S}} \sqrt{2 \cdot \mathrm{Kp}_{\perp}} \cdot \sqrt{\mathrm{t}}-\left(\frac{\mathrm{S}_{/ /}+\mathrm{S}_{\perp}}{\mathrm{S}}\right) \cdot \mathrm{Kv} \cdot \mathrm{t} \\
& \frac{\mathrm{S}_{/ /}}{\mathrm{S}} \sqrt{2 \cdot \mathrm{Kp}_{/ /}}+\frac{\mathrm{S}_{\perp}}{\mathrm{S}} \sqrt{2 \cdot \mathrm{Kp}_{\perp}}=\sqrt{2 \cdot \mathrm{Kp}}
\end{aligned}
$$




\subsection{Prise en compte des étapes d'oxydation transitoires}

L'oxyde formé pendant le chauffage avant l'atteinte de la température de palier doit aussi être pris en compte pour une meilleure précision d'analyse de la cinétique parabolique. Cet oxyde n'étant en général pas encore couvrant à l'arrivée à la température visée, l'oxydation au début du palier reste encore linéaire vis-à-vis du temps pendant une courte durée, jusqu'à ce que la couche d'oxyde externe devienne continue et que la cinétique de prise de masse devienne parabolique. La masse d'oxyde formée avant l'instauration du régime parabolique doit être prise en compte. Pour cela, l'exploitation des courbes a commencé par la détermination d'une constante d'oxydation linéaire intervenant dans l'équation (6) et représentant la cinétique linéaire observée très temporairement au début de toutes les courbes isothermes. Le gain de masse précédant le régime parabolique est la somme (7) de la partie obtenue au chauffage (indice C) et de celle résultant de l'oxydation linéaire isotherme (indice L) :

$$
\frac{\Delta \mathrm{m}}{\mathrm{S}}=\mathrm{K}_{1} \cdot \mathrm{t} \quad \text { (6) } \quad \frac{\Delta \mathrm{m}_{0}}{\mathrm{~S}}=\left(\frac{\Delta \mathrm{m}}{\mathrm{S}}\right)_{\mathrm{C}}+\left(\frac{\Delta \mathrm{m}}{\mathrm{S}}\right)_{\mathrm{L}}
$$

La quantité $\left(\frac{\Delta \mathrm{m}}{\mathrm{S}}\right)_{\mathrm{L}}$ est directement mesurée par la thermobalance, tandis que celle de l'oxydation durant le chauffage peut être estimée en supposant que la constante $\mathrm{K}_{1}$ suit une loi d'Arrhénius (8), dont la constante $K_{10}$ et l'énergie d'activation $Q$ peuvent être déduites des valeurs de $\mathrm{K}_{1}$ pour les trois températures d'essai. La prise de masse au chauffage peut être estimée au moyen d'une somme de Riemann (9) :

$$
\mathrm{K}_{\mathrm{l}}=\mathrm{K}_{\mathrm{lo}} \cdot \mathrm{e}^{\frac{-\mathrm{Q}}{\mathrm{R} \cdot \mathrm{T}}}(8) \quad\left(\frac{\Delta \mathrm{m}}{\mathrm{S}}\right)_{\mathrm{C}}=\mathrm{K}_{\mathrm{lo}_{\mathrm{lo}}} \cdot \int_{\mathrm{t}_{1}}^{\mathrm{t}_{2}} \mathrm{e}^{\frac{-\mathrm{Q}}{\mathrm{R} \cdot \mathrm{T}(\mathrm{t})}} \cdot \mathrm{dt} \approx \mathrm{K}_{\mathrm{lo}} \cdot\left(\sum_{1}^{\mathrm{N}} \mathrm{e}^{\frac{-\mathrm{Q}}{\mathrm{R} \cdot\left(273 \mathrm{~K}+\mathrm{V}_{\mathrm{c}} \cdot \mathrm{n} \cdot \Delta \mathrm{t}\right)}} \cdot \Delta \mathrm{t}\right.
$$

où $\mathrm{V}_{\mathrm{c}}$ est la vitesse de montée en température pendant le chauffage, $\Delta \mathrm{t}$ le pas d'enregistrement utilisé (40s), et $\mathrm{N}$ le nombre de ces périodes pendant le chauffage $\mathrm{N}=\frac{\mathrm{T}_{\text {palier }}-273 \mathrm{~K}}{\mathrm{~V}_{\mathrm{c}} \cdot \Delta \mathrm{t}}$.

Quand l'oxydation commence l'expression différentielle de l'équation de Wagner peut être écrite (10) en tenant compte de ce gain de masse initial (7), $\Delta \mathrm{m}_{P}$ désignant le gain de masse dû à la seule oxydation parabolique :

$$
\frac{\mathrm{d}\left(\Delta \mathrm{m}_{\mathrm{P}} / \mathrm{S}\right)}{\mathrm{dt}}=\frac{\mathrm{Kp}}{\left(\Delta \mathrm{m}_{\mathrm{P}} / \mathrm{S}+\Delta \mathrm{m}_{0} / \mathrm{S}\right)} \text { ou } \frac{\mathrm{d}\left(\Delta \mathrm{m}_{\mathrm{P}} / \mathrm{S}+\Delta \mathrm{m}_{0} / \mathrm{S}\right)}{\mathrm{dt}}=\frac{\mathrm{Kp}}{\left(\Delta \mathrm{m}_{\mathrm{P}} / \mathrm{S}+\Delta \mathrm{m}_{0} / \mathrm{S}\right)}
$$

L'intégrer conduit à l'équation (11), d'où on peut tirer Kp comme étant la pente de la droite représentant l'évolution du membre de gauche de l'équation (11), ou de (12), en fonction de t.

$$
\begin{aligned}
& \frac{1}{2}\left(\Delta \mathrm{m}_{\mathrm{P}} / \mathrm{S}+\Delta \mathrm{m}_{0} / \mathrm{S}\right)^{2}=\mathrm{Kp}\left(\mathrm{t}-\mathrm{t}_{0}\right)+\frac{1}{2}\left(\Delta \mathrm{m}_{0} / \mathrm{S}\right)^{2} \\
& \frac{1}{2}\left(\Delta \mathrm{m}_{\mathrm{P}} / \mathrm{S}+\Delta \mathrm{m}_{0} / \mathrm{S}\right)^{2}-\frac{1}{2}\left(\Delta \mathrm{m}_{0} / \mathrm{S}\right)^{2}=\mathrm{Kp}\left(\mathrm{t}-\mathrm{t}_{0}\right)
\end{aligned}
$$

Comme $\mathrm{Kp}$, la constante linéaire globale $\mathrm{K}_{1}$ est une combinaison linéaire de deux contributions, qui peuvent être séparées de la même façon :

$$
\frac{\Delta \mathrm{m}}{\mathrm{S}}(\mathrm{t})=\left(\mathrm{K}_{1}-\mathrm{Kv}\right) . \mathrm{t} \quad\left(1^{\prime}\right) \quad \frac{\Delta \mathrm{m}_{/ /}}{\mathrm{S}_{/ /}}(\mathrm{t})=\left(\mathrm{K}_{1 / /}-\mathrm{Kv}\right) . \mathrm{t} \quad\left(2^{\prime}\right) \quad \frac{\Delta \mathrm{m}_{\perp}}{\mathrm{S}_{\perp}}(\mathrm{t})=\left(\mathrm{K}_{1 \perp}-\mathrm{Kv}\right) . \mathrm{t}
$$




$$
\begin{aligned}
& \frac{\Delta \mathrm{m}}{\mathrm{S}}(\mathrm{t})=\frac{1}{\mathrm{~S}} \cdot\left(\mathrm{S}_{/ /} \cdot \frac{\Delta \mathrm{m}_{/ /}}{\mathrm{S}_{/ /}}(\mathrm{t})+\mathrm{S}_{\perp} \cdot \frac{\Delta \mathrm{m}_{\perp}}{\mathrm{S}_{\perp}}(\mathrm{t})\right)=\frac{\mathrm{S}_{/ /}}{\mathrm{S}} \cdot \mathrm{K}_{1 / /} \cdot \mathrm{t}+\frac{\mathrm{S}_{\perp}}{\mathrm{S}} \cdot \mathrm{K}_{1 \perp} \cdot \mathrm{t}-\mathrm{Kv} \cdot \mathrm{t} \\
& \frac{\mathrm{S}_{/ /}}{\mathrm{S}} \cdot \mathrm{K}_{1 / /}+\frac{\mathrm{S}_{\perp}}{\mathrm{S}} \cdot \mathrm{K}_{1 \perp}=\mathrm{K}_{1}
\end{aligned}
$$

ce qui a permis de préciser également les valeurs de constantes linéaires pour chaque orientation.

\subsection{Préparation des échantillons pour examens métallographiques}

Les échantillons pour examen de microstructure ont été enrobés à l'aide d'une résine Araldite CY230 additionnée d'un durcisseur Escil HY956. Leur polissage d'ébauche a été réalisé au papier SiC de gradation 80 à 1200 sous courant d'eau, et celui de finition au feutre avec pâte diamantée $6 \mu \mathrm{m}$ DP-Suspension P de Struers). Les microstructures ont été observées au Microscope Electronique à Balayage (MEB) de type Philips XL30, sous une tension d'accélération de 20kV, et essentiellement en mode électrons rétrodiffusés (BSE). L'analyse chimique de chaque alliage a été contrôlée au MEB avec son dispositif de Spectrométrie de Dispersion d'Energie (EDS). Au sujet du carbone qui ne peut pas être analysé de cette manière, l'évaluation métallographique de la densité des réseaux de carbures a permis de vérifier que les teneurs massiques visées ont été à peu près respectées.

La préparation des échantillons oxydés en vue de l'examen des morceaux de couche d'oxyde extérieur et des zones superficielles affectées par l'oxydation a inclus des étapes supplémentaires en vue de préserver la couche d'oxyde lorsque le refroidissement post-essai ne l'a pas totalement fait quitter l'échantillon. Un dépôt d'or a tout d'abord été fait par pulvérisation cathodique pour rendre l'extérieur de l'échantillon électriquement conducteur. Cela a permis ensuite de déposer de façon électrolytique une couche de nickel couvrante et épaisse pour protéger l'oxyde extérieur lors de la découpe de l'échantillon. Ces échantillons ont fait l'objet d'acquisition de profils de concentration au travers de la zone affectée par l'oxydation, au moyen d'une microsonde de Castaing Cameca SX100 en spectrométrie de dispersion des longueurs d'onde (WDS).

\section{RESULTATS}

\subsection{Microstructures des alliages}

Le tableau I présente les compositions chimiques obtenues pour les deux alliages (mesures EDS réalisées avec le MEB) et la figure 3 illustre les microstructures des deux alliages à l'état brut de coulée. Tous deux sont constitués d'une matrice dendritique de nickel contenant du chrome et de carbone en solution solide, et de carbures de chrome formant un réseau interdendritique. Ces carbures sont naturellement plus nombreux dans l'alliage à $0,8 \% \mathrm{C}$ que dans celui à $0,2 \% \mathrm{C}$.

Tableau I. Compositions chimiques obtenues pour les deux alliages

\begin{tabular}{|c|c|c|c|}
\hline Teneurs massiques & $\begin{array}{c}\mathrm{Ni} \\
(\%)\end{array}$ & $\begin{array}{c}\mathrm{Cr} \\
(\%)\end{array}$ & $\begin{array}{c}\mathrm{C} \\
(\%)\end{array}$ \\
\hline Ni30Cr0,2C & $70,5 \pm 0,2$ & $29,5 \pm 0,2$ & $\cong 0,2$ \\
\hline Ni30Cr0,8C & $70,8 \pm 0,7$ & $29,2 \pm 0,7$ & $\cong 0,8$ \\
\hline
\end{tabular}



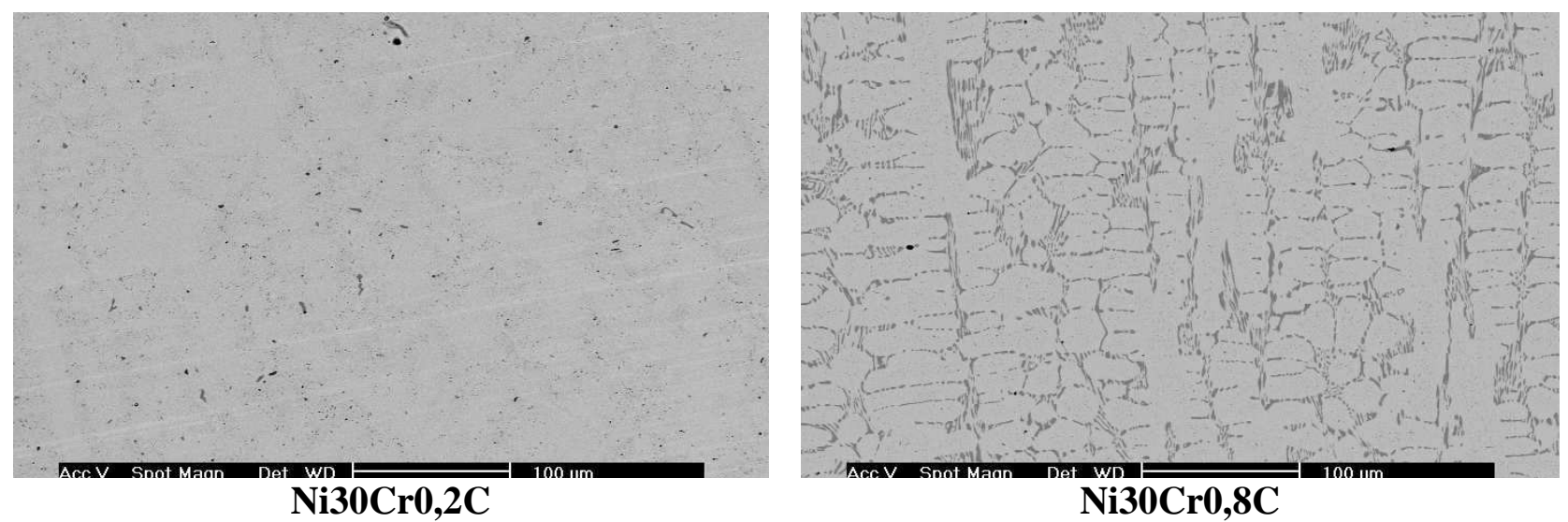

Figure 3. Microstructures des deux alliages base nickel étudiés, à l'état brut de coulée

\subsection{Résultats thermogravimétriques}

Pour chacun des deux alliages, les essais thermogravimétriques d'oxydation ont été réalisés pour les deux types d'orientation d'échantillon et pour les trois températures. Les courbes de prise de masse brutes obtenues sont toutes d'allure parabolique, après une courte oxydation linéaire en tout début de palier. Deux d'entre elles présentent des sauts de prise de masse, qui heureusement sont d'assez faible amplitude ce qui n'a pas empêché la détermination des constantes paraboliques. Les courbes obtenues pour les deux températures extrêmes sont présentées dans la figure 4 à titre d'exemples, et on peut déjà voir que l'orientation de la surface par rapport aux dendrites sousjacentes semble avoir en effet une influence sur la cinétique de prise de masse.

Les constantes $\mathrm{K}_{1}$ ont été déterminées sur le début linéaire des courbes. Leurs valeurs sont données dans le tableau II. Pour chaque couple alliage-orientation la constante linéaire globale augmente logiquement avec la température. Pour l'alliage le plus riche en carbone la constante paraît plus élevée pour un échantillon de surface majoritairement perpendiculaire aux dendrites que pour l'autre type d'échantillon, et ce à toutes températures. Cependant, les deux valeurs obtenues à $1200^{\circ} \mathrm{C}$ sont très proches l'une de l'autre. Après découplage par résolution du couple d'équations (5'), les mêmes remarques peuvent être faites, de façon accentuée. Pour l'alliage le moins riche en carbone la comparaison est plus complexe : l'oxydation linéaire préliminaire paraît légèrement plus rapide pour l'orientation perpendiculaire à $1000^{\circ} \mathrm{C}$ tandis que cela deviendrait l'inverse à $1200^{\circ} \mathrm{C}$. A la température intermédiaire les deux vitesses sont égales. Pour cet alliage aussi, le découplage amplifie les différences observées. Dans le cas de l'alliage Ni30Cr0,2C la constante d'oxydation linéaire semble suivre une loi d'Arrhénius, comme le montre la figure 5. Pour l'autre alliage, pour lequel les trois points sont moins alignés, surtout dans le cas de l'orientation parallèle, ce serait moins vrai. Malgré qu'il eût été préférable de disposer de résultats pour plus de trois températures différentes, il a été fait l'hypothèse que la constante linéaire suit dans tous les cas une loi d'Arrhénius, même quand les points ne sont pas vraiment alignés. Il est en effet préférable de disposer d'une valeur approchée de l'épaisseur d'oxyde préexistant au début du palier pour la prendre en compte dans l'analyse des courbes, plutôt que de la prendre nulle. Ainsi des énergies d'activation ont été estimées pour les quatre combinaisons alliage <-> orientation (dernière colonne du tableau II), et les équations de régression linéaire ont permis d'estimer les gains de masse préexistants pour tous les essais thermogravimétriques en vue d'une meilleure précision des constantes paraboliques.

Pour tous les essais il a été possible de déterminer la constante parabolique du fait que la quantité $\left(\Delta \mathrm{m}_{\mathrm{p}} / \mathrm{S}+\Delta \mathrm{m}_{\mathrm{o}} / \mathrm{S}\right)^{2}-\left(\Delta \mathrm{m}_{\mathrm{o}} / \mathrm{S}\right)^{2}$ variait manifestement bien de façon linéaire en fonction 

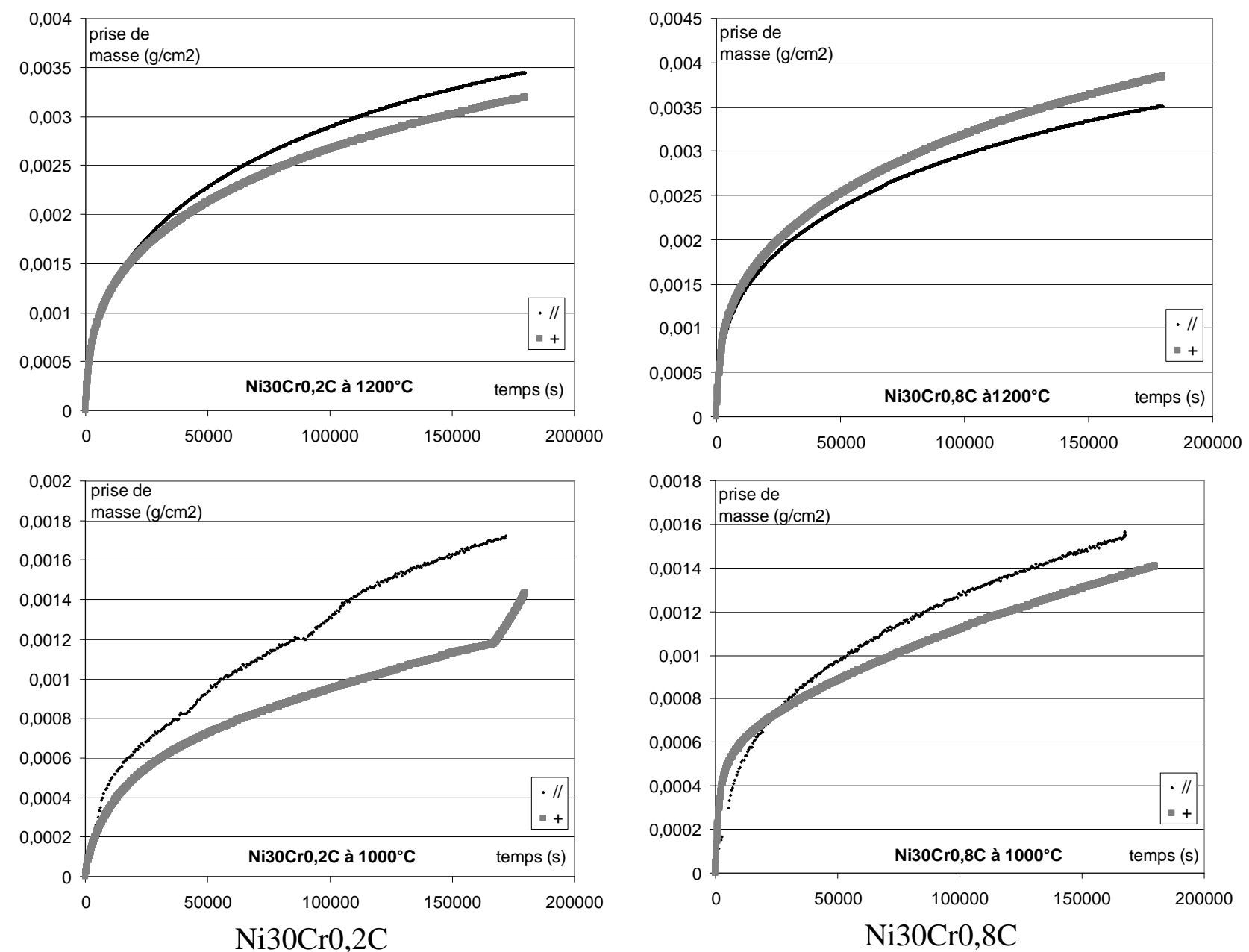

Figure 4. Quelques-unes des courbes thermogravimétriques obtenues (trait fin : parallèle aux dendrites, trait épais : perpendiculaire aux dendrites)

Tableau II. Valeurs des constantes linéaires d'oxydation globales et découplées (en gras)

\begin{tabular}{|c|c|c|c|c|c|}
\hline \multicolumn{2}{|c|}{$\mathrm{K}_{1}\left(10^{-8} \mathrm{~g} \cdot \mathrm{cm}^{-2} \cdot \mathrm{s}^{-1}\right)$} & $1000^{\circ} \mathrm{C}$ & $1100^{\circ} \mathrm{C}$ & $1200^{\circ} \mathrm{C}$ & Energies d'activation $\left(\mathrm{kJ} . \mathrm{mol}^{-1}\right)$ \\
\hline \multirow{4}{*}{$\mathrm{Ni} 30 \mathrm{Cr} 0,2 \mathrm{C}$} & gl. & $5,9 \pm 0,8$ & $22 \pm 2,9$ & $75 \pm 10$ & \multirow[b]{2}{*}{ estimée à env. $\mathbf{2 1 0}$} \\
\hline & $/ /$ & $\mathbf{5 , 7} \pm 0,7$ & $22 \pm 2,9$ & $86 \pm 11$ & \\
\hline & gl. & $7,2 \pm 0,9$ & $22 \pm 2,9$ & $57 \pm 7,4$ & \multirow[b]{2}{*}{ estimée à env. 125} \\
\hline & $\perp$ & $\mathbf{7 , 9} \pm 1,0$ & $22 \pm 2,9$ & $40 \pm 5,2$ & \\
\hline \multirow{4}{*}{$\mathrm{Ni} 30 \mathrm{Cr} 0,8 \mathrm{C}$} & gl. & $5,6 \pm 0,7$ & $11 \pm 1,4$ & $62 \pm 8,1$ & \multirow[b]{2}{*}{ estimée à env. $\mathbf{2 3 0}$} \\
\hline & // & $\mathbf{3 , 2} \pm 0,4$ & $\mathbf{6 , 9} \pm 0,9$ & $\mathbf{6 0} \pm 7,8$ & \\
\hline & gl. & $20 \pm 2,6$ & $39 \pm 5,1$ & $64 \pm 8,3$ & \multirow[b]{2}{*}{ estimée à env. 80} \\
\hline & $\perp$ & $\mathbf{2 6} \pm 3,4$ & $\mathbf{5 5} \pm 7,2$ & $\mathbf{6 6} \pm 8,6$ & \\
\hline
\end{tabular}

de $\mathrm{t}_{-} \mathrm{t}_{0}$ sur une bonne partie de la courbe. Ceci a conduit aux valeurs des constantes paraboliques englobant les participations des deux types de surfaces de chaque échantillon, puis aux constantes spécifiques aux deux orientations après découplage par résolution du système de deux équations de type (5). Comme pour les constantes linéaires les différences déjà constatées entre les valeurs de Kp globales des deux types d'échantillons se sont trouvées amplifiées par le découplage, avec comme conséquence une différentiation plus marquée entre surface parallèle et surface perpendiculaire aux dendrites (tableau III). Pour l'alliage Ni30Cr0,2C les constantes Kp correspondant à l'orientation parallèle sont plus élevées que celles obtenues pour le cas perpendiculaire, notamment à $1000^{\circ} \mathrm{C}$, et

Contact : patrice.berthod@ centraliens-lille.org 

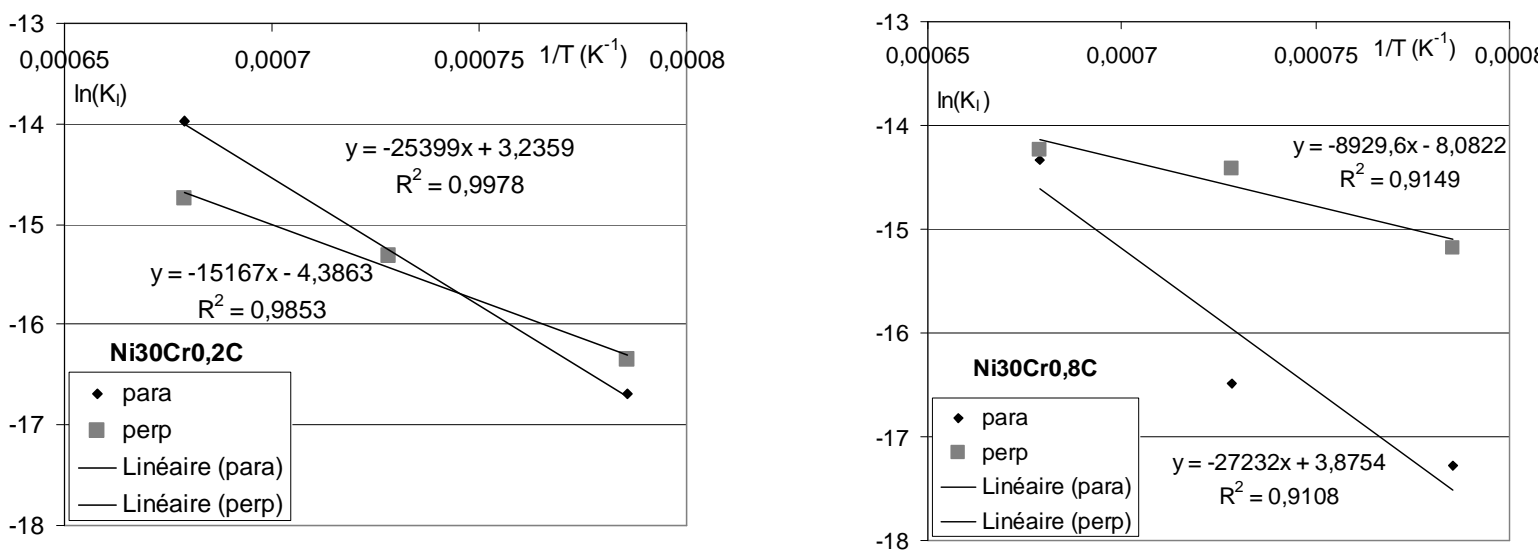

Figure 5. Evolution des constantes linéaires $\mathrm{K}_{1}$ avec la température, dans une représentation d'Arrhénius pour les deux alliages $\left(\ln \left[\mathrm{K}_{1} /\left(1 \mathrm{~g} \cdot \mathrm{cm}^{-2} \cdot \mathrm{s}^{-1}\right)\right]=\mathrm{f}\left(\mathrm{T}(\mathrm{K})^{-1}\right)\right.$

Tableau III. Valeurs des constantes paraboliques d'oxydation globales et découplées (en gras)

\begin{tabular}{|c|c|c|c|c|c|}
\hline $\mathrm{Kp}\left(10^{-12} \mathrm{~g}^{2} \cdot \mathrm{cm}^{-4} \cdot \mathrm{s}^{-1}\right)$ & $1000^{\circ} \mathrm{C}$ & $1100^{\circ} \mathrm{C}$ & $1200^{\circ} \mathrm{C}$ & Energie d'activation $\left(\mathrm{kJ} \cdot \mathrm{mol}^{-1}\right)$ \\
\hline \multirow{3}{*}{ Ni30Cr0,2C } & gl. & $11 \pm 1,4$ & $41 \pm 5,3$ & $115 \pm 15$ & \\
& $/ /$ & $\mathbf{1 2} \pm 1,6$ & $\mathbf{4 3} \pm 5,6$ & $\mathbf{1 1 8} \pm 15$ & estimée à env. 180 \\
\cline { 2 - 6 } & gl. & $6,9 \pm 0,9$ & $36 \pm 4,7$ & $109 \pm 14$ & \\
& $\perp$ & $\mathbf{4 , 9} \pm 0,8$ & $\mathbf{3 3} \pm 4,3$ & $\mathbf{1 0 3} \pm 13$ & estimée à env. 240 \\
\hline \multirow{3}{*}{ Ni30Cr0,8C } & gl. & $9,7 \pm 1,3$ & $50 \pm 6,5$ & $113 \pm 15$ & \\
& $/ /$ & $\mathbf{1 0} \pm 1,3$ & $\mathbf{4 8} \pm 6,3$ & $\mathbf{9 9} \pm 13$ & estimée à env. $\mathbf{1 8 0}$ \\
\cline { 2 - 6 } & gl. & $8,0 \pm 1,1$ & $62 \pm 8,1$ & $129 \pm 17$ & \\
& $\perp$ & $\mathbf{7 , 4} \pm 1,0$ & $\mathbf{6 9} \pm 9,0$ & $\mathbf{1 4 2} \pm 19$ & estimée à env. 230 \\
\hline
\end{tabular}

toutes deux augmentent logiquement avec la température, en suivant manifestement une loi d'Arrhénius (figure 6). Par contre c'est généralement l'inverse pour l'alliage Ni30Cr0,8C, et l'alignement des points dans la représentation d'Arrhénius des Kp découplées est moins bon pour cet alliage (figure 6). Le mauvais alignement des points obtenus, qui plus est que pour trois températures seulement, ne permet pas d'affirmer que Kp suive vraiment une loi d'Arrhénius, et si
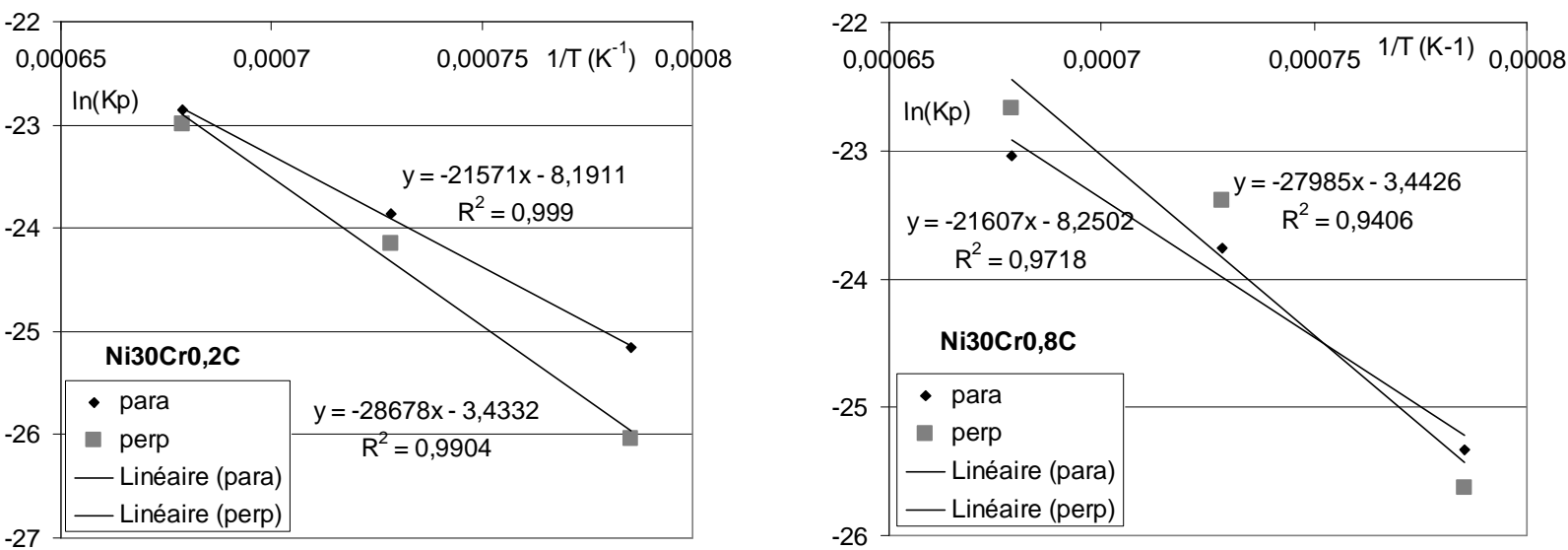

Figure 6. Evolution des constantes paraboliques $\mathrm{Kp}$ avec la température, dans une représentation d'Arrhénius pour les deux alliages $\left(\ln \left[\mathrm{Kp} /\left(1 \mathrm{~g}^{2} \cdot \mathrm{cm}^{-4} \cdot \mathrm{s}^{-1}\right)\right]=\mathrm{f}\left(\mathrm{T}(\mathrm{K})^{-1}\right)\right.$ 
c'était le cas de déterminer une valeur précise d'énergie d'activation. Cependant, afin de permettre tout de même une comparaison entre les deux orientations pour cet alliage aussi, deux énergies d'activation supposées ont été estimées. Moyennant ce qui précède, la pente de la droite de régression serait pour les deux alliages plus forte pour l'orientation perpendiculaire que pour l'orientation parallèle, et donc les énergies d'activation seraient pour les surfaces parallèles $\left(Q_{/ /}\right.$de l'ordre de 230-240 $\left.\mathrm{kJ} \cdot \mathrm{mol}^{-1}\right)$ supérieures à celles pour les surfaces perpendiculaires $(\mathrm{Q} \perp$ de l'ordre de $180 \mathrm{~kJ} \cdot \mathrm{mol}^{-1}$ ).

\subsection{Caractérisation de la surface oxydée}

Après les essais thermogravimétriques tous les échantillons ont fait l'objet d'observations métallographiques, en microscopie optique et au MEB (figures 7 et 8). Dans tous les cas une couche d'oxyde $\mathrm{Cr}_{2} \mathrm{O}_{3}$ couvrante s'est développée sur la surface extérieure. Malheureusement cette dernière s'est généralement détachée de l'échantillon au cours du refroidissement si bien que, dans la plupart des cas, seules quelques parties d'oxyde sont restées ce qui a toutefois permis de les observer et d'en estimer l'épaisseur.

Pour les deux alliages et pour les trois températures les carbures de chrome initialement présents jusqu'à la surface extérieure ont disparu sur une certaine profondeur à partir de cette dernière. Cette profondeur décarburée augmente avec la température d'essai mais dépend aussi de l'alliage concerné. Elle contient parfois des trous ou vides, plus ou moins présents suivant l'alliage et suivant la température.

Dans certains cas, et notamment aux deux plus basses températures, on peut aussi observer des modifications des carbures à la frontière séparant la zone décarburée du reste de l'alliage. Dans cette zone intermédiaire les carbures semblent avoir subi des modifications de taille (plus grossiers), de stoechiométrie (plus sombres vus au MEB en mode BSE), ou certains, de forme aciculaire, paraissent avoir précipité à l'état solide au cœur de la matrice. Le tableau IV synthétise l'ensemble de ces observations pour les deux alliages, les deux orientations et les trois températures. Les vides dans la zone décarburée existent en plus grande quantité pour l'alliage Ni30Cr0,8C que pour l'autre alliage, et ils sont aussi dépendants de la température. L'intervalle de température pour lequel ils existent est plus bas pour l'alliage $\mathrm{Ni30Cr} 0,2 \mathrm{C}$ que pour l'alliage $\mathrm{Ni30Cr} 0,8 \mathrm{C}$, et pour ce deuxième alliage, il est plus élevé pour l'orientation perpendiculaire que pour l'orientation parallèle. Les phénomènes de grossissement ou «de noircissement» des carbures, qui concernent surtout les températures de 1000 et $1100^{\circ} \mathrm{C}$, sont plus marqués à $1000^{\circ} \mathrm{C}$ pour l'alliage à $0,8 \% \mathrm{C}$ que pour celui à $0,2 \% \mathrm{C}$ (alors que ce serait plutôt l'inverse pour $1100^{\circ} \mathrm{C}$ ), et, pour les deux alliages, plutôt pour l'orientation parallèle que pour l'orientation perpendiculaire.

Le tableau $V$ présente les valeurs de l'épaisseur de la couche de chromine extérieure et de la profondeur de la zone décarburée. La hiérarchie des épaisseurs de la couche de chromine est en accord avec celle des constantes paraboliques puisque plus $\mathrm{Kp}$ est élevée, plus l'épaisseur mesurée

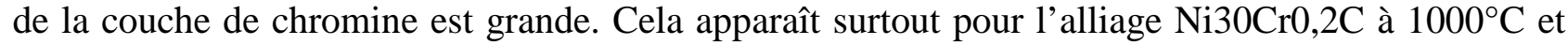
pour l'alliage $\mathrm{Ni} 30 \mathrm{Cr} 0,8 \mathrm{C}$ à $1200^{\circ} \mathrm{C}$ puisque d'une part les valeurs de $\mathrm{Kp}$ et d'autre part les valeurs d'épaisseur de chromine sont plutôt groupées dans tous les autres cas. La profondeur décarburée augmente avec la température et, pour les deux plus hautes températures, elle diminue quand la densité initiale des carbures augmente. Cela dépend aussi de l'orientation dendritique puisque la profondeur décarburée serait plus grande pour l'orientation parallèle que pour l'orientation perpendiculaire aux deux plus basses températures. Par contre, il n'y a pas d'influence de l'orientation sur la profondeur décarburée à $1200^{\circ} \mathrm{C}$, quelque soit l'alliage. 


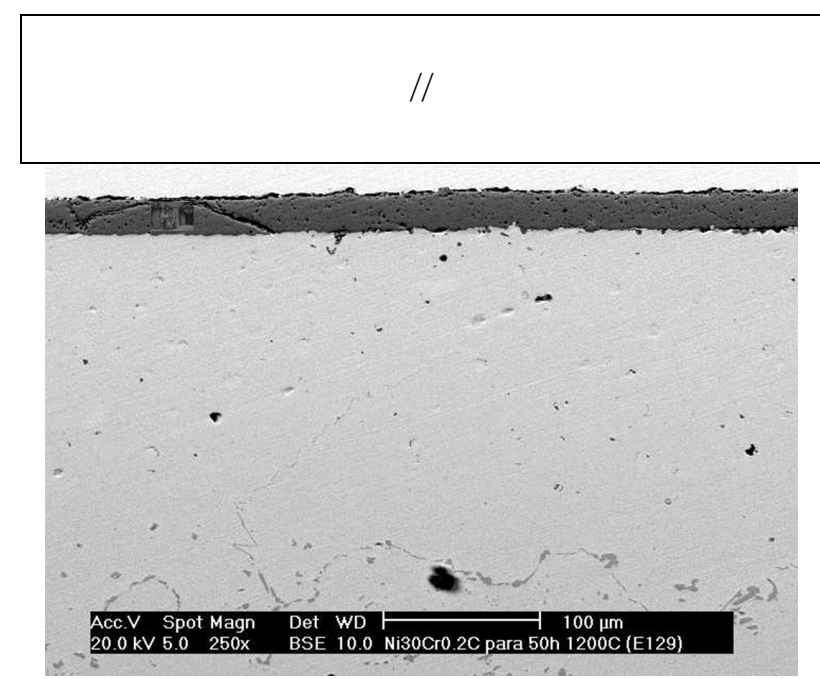

$1200^{\circ} \mathrm{C}$
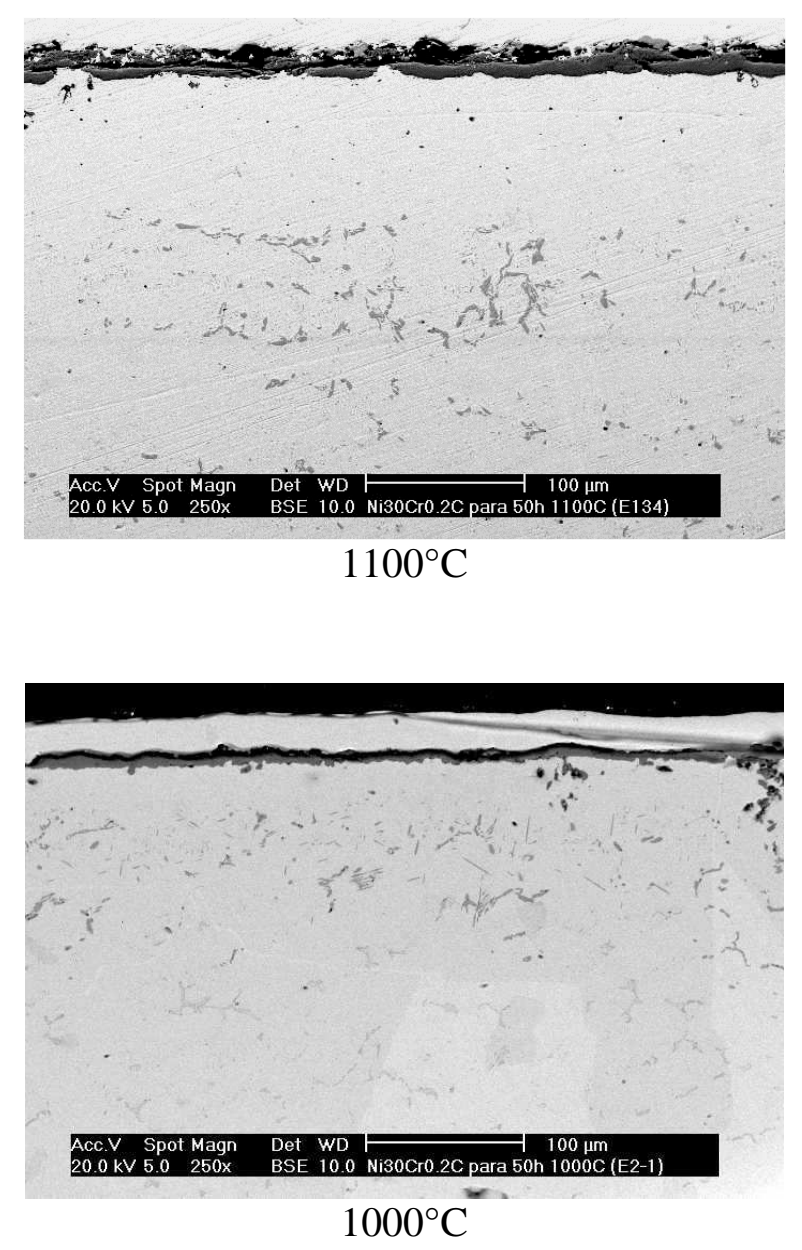
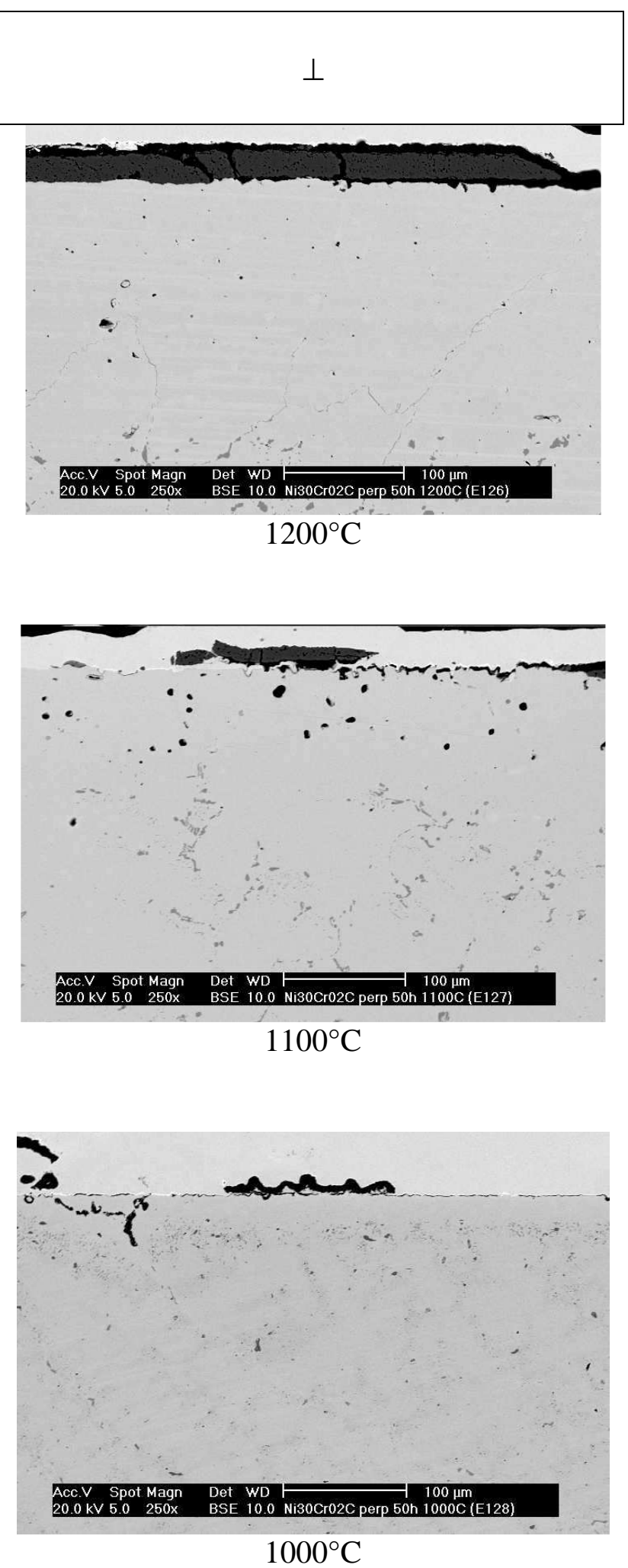

Figure 7. Etat de la surface oxydée de l'alliage Ni30Cr0,2C pour les orientations parallèle (gauche) et perpendiculaire (droite) 

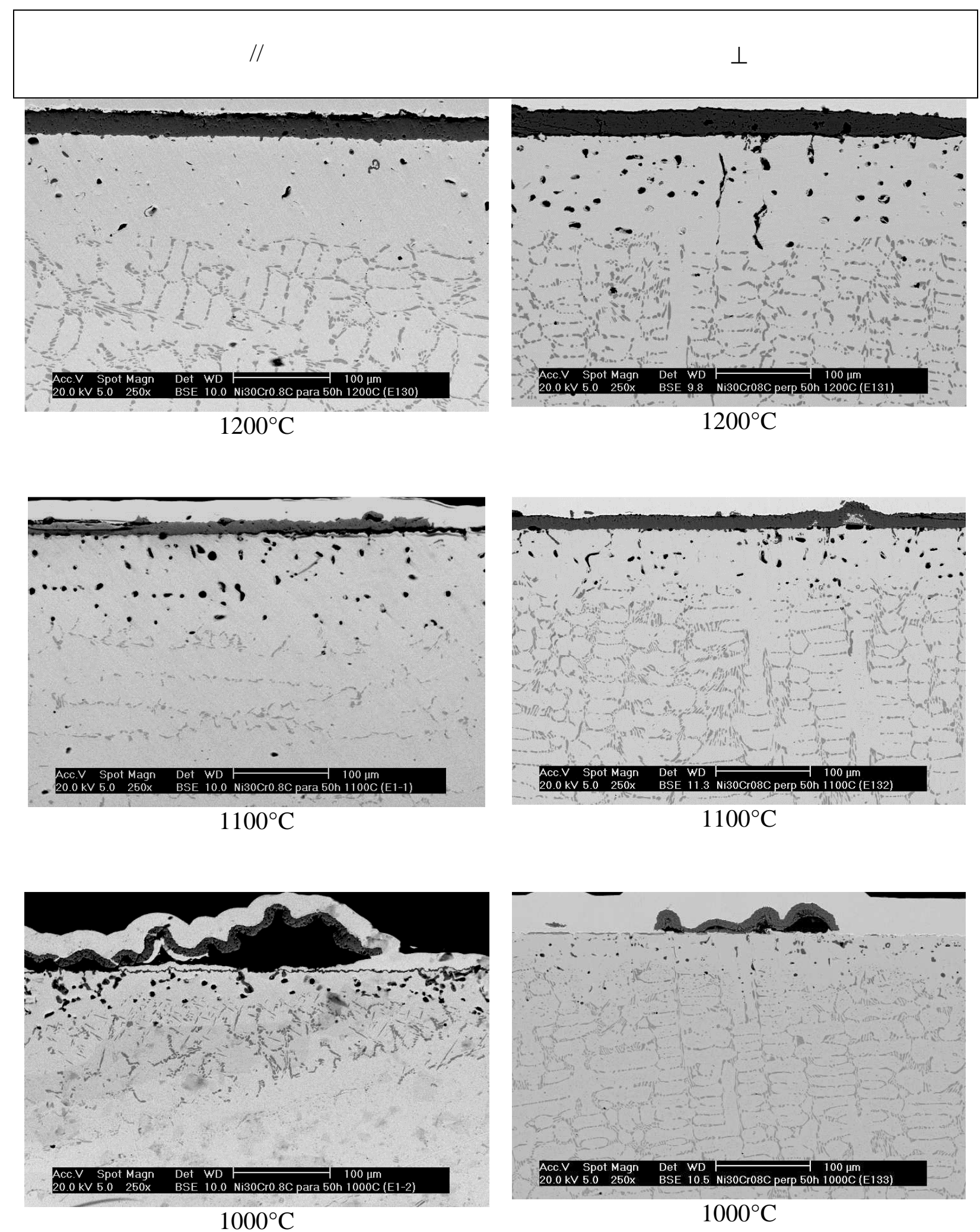

Figure 8. Etat de la surface oxydée de l'alliage Ni30Cr0,8C pour les orientations parallèle (gauche) et perpendiculaire (droite) 
Tableau IV. Description qualitative de l'état de la zone affectée par l'oxydation

\begin{tabular}{|c|c|c|c|c|}
\hline \multirow[t]{2}{*}{ température } & \multicolumn{2}{|c|}{$\mathrm{Ni} 30 \mathrm{Cr} 0,2 \mathrm{C}$} & \multicolumn{2}{|c|}{ Ni30Cr0,8C } \\
\hline & // & $\perp$ & // & $\perp$ \\
\hline \multirow[b]{2}{*}{$1200^{\circ} \mathrm{C}$} & & & o & 000 \\
\hline & & & & \\
\hline \multirow[b]{2}{*}{$1100^{\circ} \mathrm{C}$} & & $\mathrm{O}$ & 000 & 00 \\
\hline & c c & c & & \\
\hline \multirow{3}{*}{$1000^{\circ} \mathrm{C}$} & $\mathrm{O}$ & & $\mathrm{OO}$ & $\mathrm{O}$ \\
\hline & c c & & c c c & c \\
\hline & X X & $X$ & X X X & \\
\hline \multicolumn{5}{|c|}{$\begin{array}{c}\mathbf{c}: \text { carbures plus grossiers ou plus sombres } \\
\text { X : carbures aciculaires }\end{array}$} \\
\hline
\end{tabular}

Tableau V. Epaisseurs de la couche de chromine extérieure et profondeurs décarburées

\begin{tabular}{|c|c|c|c|c|}
\hline $\begin{array}{c}\text { Epaisseur } \\
\text { d'oxyde }(\mu \mathrm{m}) \\
+/-1 \text { écart-type }\end{array}$ & \multicolumn{2}{|c|}{ Ni30Cr0,2C } & \multicolumn{2}{c|}{ Ni30Cr0,8C } \\
\hline $\begin{array}{c}\text { Profondeur } \\
\text { décarburée }(\mu \mathrm{m}) \\
+/-1 \text { écart-type }\end{array}$ & $/ /$ & $\perp$ & $1 /$ & $\perp$ \\
\hline \multirow{2}{*}{$1200^{\circ} \mathrm{C}$} & $20,0 \pm 1,2$ & $22,9 \pm 1,0$ & $21,6 \pm 1,0$ & $23,9 \pm 1,0$ \\
\cline { 2 - 5 } & $206 \pm 8$ & $205 \pm 10$ & $105 \pm 3$ & $106 \pm 6$ \\
\hline \multirow{2}{*}{$1100^{\circ} \mathrm{C}$} & $10,4 \pm 1,8$ & $11,0 \pm 1,8$ & $12,1 \pm 2,3$ & $13,7 \pm 1,0$ \\
\cline { 2 - 5 } & $86,5 \pm 4,8$ & $85,4 \pm 7,9$ & $85,4 \pm 4,8$ & $56,7 \pm 7,8$ \\
\hline \multirow{2}{*}{$1000^{\circ} \mathrm{C}$} & $9,7 \pm 1,0$ & $5,1 \pm 0,7$ & $7,6 \pm 1,0$ & $8,8 \pm 1,3$ \\
\cline { 2 - 5 } & $27,1 \pm 3,9$ & $20,4 \pm 1,4$ & $22,4 \pm 3,3$ & $27,1 \pm 3,6$ \\
\hline
\end{tabular}

\subsection{Profils de concentration en chrome au travers de la zone décarburée des alliages oxydés}

Des profils de concentration perpendiculaires à la surface extérieure et traversant la zone affectée par l'oxydation ont été réalisés sur la face principale de chaque échantillon oxydé, à l'aide d'une microsonde de Castaing Cameca SX100, à raison de deux profils par échantillon. Les profils sont en une ou deux parties d'évolution linéaire de la teneur massique en chrome, ceci suivant l'alliage et suivant la température de l'essai d'oxydation. Aucune vraie différence n'existe entre les deux orientations pour l'alliage $\mathrm{Ni30Cr} 0,2 \mathrm{C}$, quelque soit la température (figure 9). Par contre, pour l'alliage Ni30Cr0,8C, s'il n'y a pas non plus de différence entre les deux orientations pour $1200^{\circ} \mathrm{C}$, une différence commence à apparaître à $1100^{\circ} \mathrm{C}$ et elle devient manifeste à $1000^{\circ} \mathrm{C}$ (figure 10 ).

Le tableau VI présente les gradients moyens et les étendues de ces derniers. Tous les gradients voient leur norme diminuer quand la température augmente, mais leur valeur varie aussi

Contact : patrice.berthod@ centraliens-lille.org 
Ni-30Cr0,2C après $50 \mathrm{~h}$ à $1200^{\circ} \mathrm{C}$ (orientation parallèle)

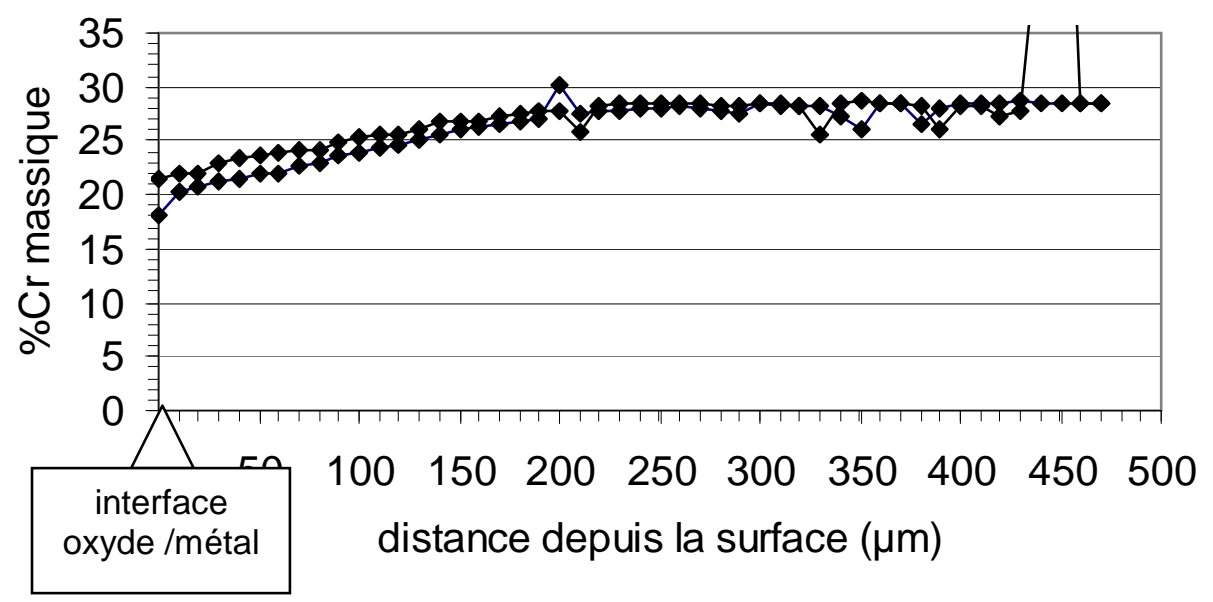

$\mathrm{Ni}-30 \mathrm{Cr} 0,2 \mathrm{C}$ après $50 \mathrm{~h}$ à $1200^{\circ} \mathrm{C}$ (orientation perpendiculaire aux dendrites)

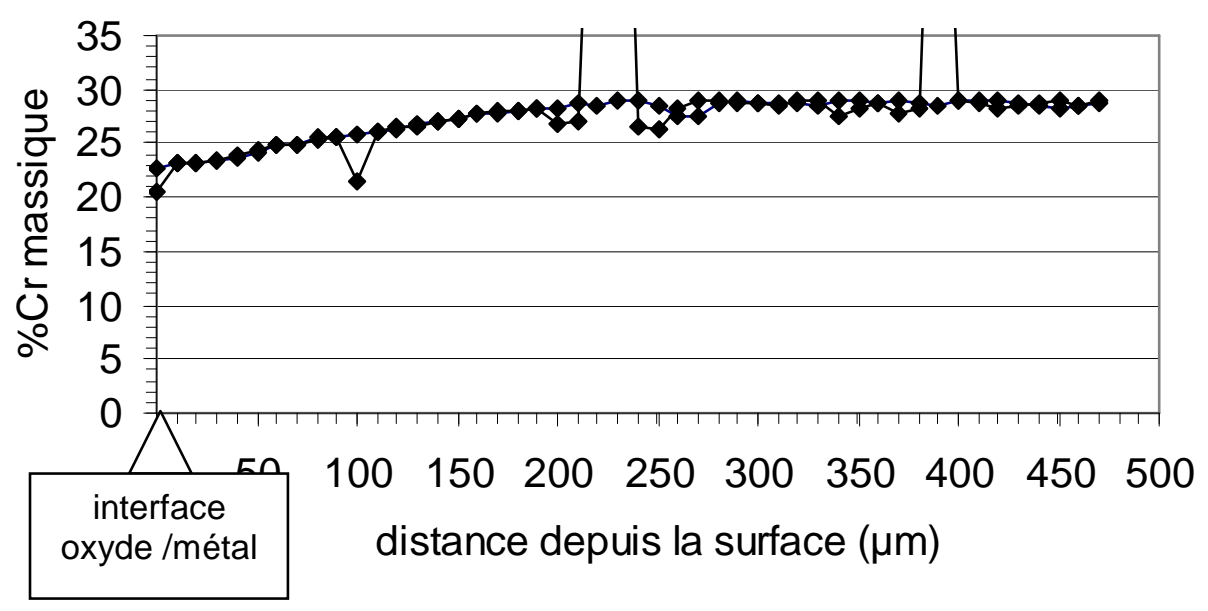

Figure 9. Profils de concentration du chrome pour l'alliage $\mathrm{Ni30Cr} 0,2 \mathrm{C}$ oxydé à $1200^{\circ} \mathrm{C}$ : pas de différence sensible entre les deux orientations dendritiques

avec l'alliage et l'orientation dendritique. Dans le cas de l'alliage à $0,2 \% \mathrm{C}$, l'oxydation à $1000^{\circ} \mathrm{C}$ a conduit à deux gradients successifs (par contre un seul gradient pour 1100 et $1200^{\circ} \mathrm{C}$ ), l'étendue du premier correspondant à l'épaisseur décarburée (tableau $V$ ). Ce gradient est plus élevé pour l'orientation perpendiculaire que pour l'orientation parallèle. L'unique gradient observé pour $1100^{\circ} \mathrm{C}$ est au contraire plus faible pour l'orientation perpendiculaire que pour l'autre orientation, tandis qu'il n'y a plus de différence à $1200^{\circ} \mathrm{C}$. Pour l'alliage à $0,8 \% \mathrm{C}$, l'existence de deux gradients, déjà vraie pour $1000^{\circ} \mathrm{C}$, persiste jusqu'à $1200^{\circ} \mathrm{C}$. Le premier gradient, le plus fort, correspond toujours à la zone décarburée, et il est à toutes températures plus élevé pour l'orientation parallèle que pour l'orientation perpendiculaire (une tendance non suivie en revanche par le second gradient quand ce dernier est faible, soit à 1100 et $1200^{\circ} \mathrm{C}$ ). A part dans le cas de l'orientation parallèle pour $1000^{\circ} \mathrm{C}$, le premier gradient (ou unique gradient suivant les cas) est toujours plus faible pour l'alliage Ni30Cr0,8C que pour l'alliage Ni30Cr0,2C. 
Ni-30Cro, $8 \mathrm{C}$ après $50 \mathrm{~h}$ à $1000^{\circ} \mathrm{C}$ (surface parallèle aux dendrites)

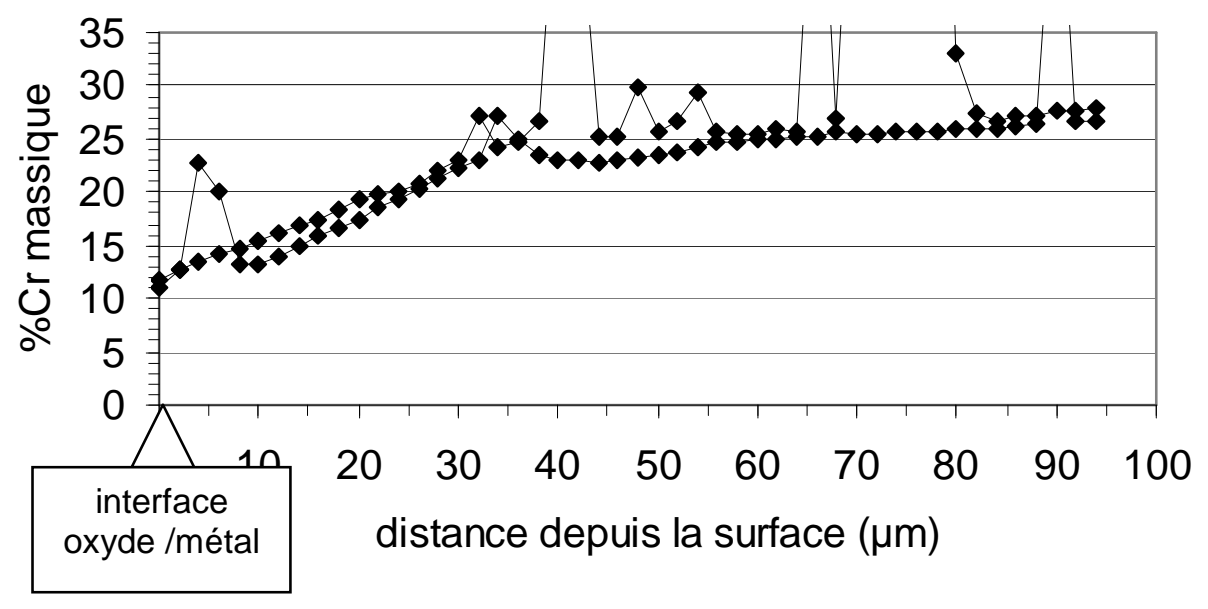

$\mathrm{Ni}-30 \mathrm{Cr} 0,8 \mathrm{C}$ après $50 \mathrm{~h}$ à $1000^{\circ} \mathrm{C}$ (orientation perpendiculaire aux dendrites)

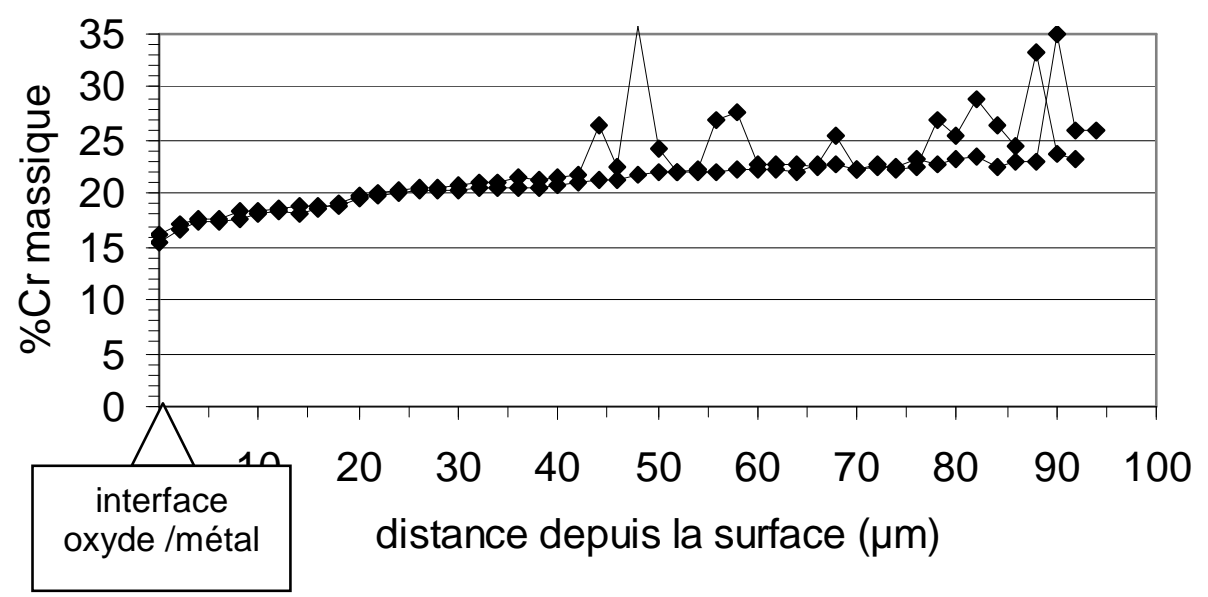

Figure 10. Profils de concentration du chrome pour l'alliage $\mathrm{Ni} 30 \mathrm{Cr} 0,8 \mathrm{C}$ oxydé à $1000^{\circ} \mathrm{C}$ : différence importante entre les deux orientations dendritiques

\subsection{Coefficients de diffusion du chrome au travers de la zone décarburée des alliages oxydés}

Connaissant à la fois les gradients en chrome et les vitesses locales d'oxydation $\left(\mathrm{Kp}_{/ /} \mathrm{ou} \perp\right)$, il est possible d'estimer les valeurs des coefficients de diffusion du chrome dans la zone décarburée (qui est faite d'alliage $\mathrm{Ni}-(<30 \%) \mathrm{Cr}$ et dont les joints de grains sont orientés de manière spécifique). On peut en effet écrire l'équation (13) en exprimant la vitesse de consommation de chrome par le phénomène d'oxydation en toute fin d'essai thermogravimétrique (au bout des 50 heures) :

$$
\left.\frac{\mathrm{d}\left(\frac{\mathrm{n}_{\mathrm{Cr}}}{\mathrm{S}}\right)}{\mathrm{dt}}\right|_{\text {fin }}=\mathrm{D}_{\mathrm{Cr}} \cdot\|\overrightarrow{\mathrm{grad}}[\mathrm{Cr}]\|
$$


Tableau VI. Gradients moyens de concentration en chrome perpendiculairement à la surface et au travers de la zone déchromisée

\begin{tabular}{|c|c|c|c|c|c|c|c|}
\hline \multirow{2}{*}{\multicolumn{2}{|c|}{$\begin{array}{l}\text { Gradients } \mathrm{Cr} \\
\left(\%_{\text {mass }} / \mu \mathrm{m}\right)\end{array}$}} & \multicolumn{2}{|c|}{$1000^{\circ} \mathrm{C}$} & \multicolumn{2}{|c|}{$1100^{\circ} \mathrm{C}$} & \multicolumn{2}{|c|}{$1200^{\circ} \mathrm{C}$} \\
\hline & & external & internal & external & internal & external & internal \\
\hline \multirow{2}{*}{$\begin{array}{c}\mathrm{Ni} 30 \mathrm{Cr} \\
0,2 \mathrm{C}\end{array}$} & $/ /$ & $\begin{array}{l}0,230 \\
\text { sur } \\
28 \mu \mathrm{m}\end{array}$ & $\begin{array}{c}0,028 \\
\text { sur plus de } \\
70 \mu \mathrm{m}\end{array}$ & $\begin{array}{l}0,191 \\
\text { sur } \\
90 \mu \mathrm{m}\end{array}$ & trop faible & $\begin{array}{l}0,035 \\
\text { sur } \\
220 \mu \mathrm{m}\end{array}$ & trop faible \\
\hline & $\perp$ & $\begin{array}{l}0,339 \\
\text { sur } \\
17 \mu \mathrm{m}\end{array}$ & $\begin{array}{c}0,014 \\
\text { sur } \\
35 \mu \mathrm{m}\end{array}$ & $\begin{array}{l}0,118 \\
\text { sur } \\
85 \mu \mathrm{m}\end{array}$ & trop faible & $\begin{array}{c}0,032 \\
\text { sur } \\
210 \mu \mathrm{m}\end{array}$ & trop faible \\
\hline \multirow{2}{*}{$\begin{array}{c}\mathrm{Ni} 30 \mathrm{Cr} \\
0,8 \mathrm{C}\end{array}$} & // & $\begin{array}{c}0,390 \\
\text { sur } \\
33 \mu \mathrm{m}\end{array}$ & $\begin{array}{c}0,063 \\
\text { sur plus de } \\
70 \mu \mathrm{m}\end{array}$ & $\begin{array}{c}0,069 \\
\text { sur } \\
90 \mu \mathrm{m}\end{array}$ & $\begin{array}{c}0,009 \\
\text { sur } \\
135 \mu \mathrm{m}\end{array}$ & $\begin{array}{c}0,027 \\
\text { sur } \\
110 \mu \mathrm{m}\end{array}$ & $\begin{array}{l}0,005 \\
\text { sur } \\
290 \mu \mathrm{m}\end{array}$ \\
\hline & $\perp$ & $\begin{array}{c}0,148 \\
\text { sur } \\
26 \mu \mathrm{m}\end{array}$ & $\begin{array}{c}0,050 \\
\text { sur plus de } \\
70 \mu \mathrm{m}\end{array}$ & $\begin{array}{c}0,040 \\
\text { sur } \\
65 \mu \mathrm{m}\end{array}$ & $\begin{array}{c}0,021 \\
\text { sur } \\
190 \mu \mathrm{m}\end{array}$ & $\begin{array}{c}0,015 \\
\text { sur } \\
120 \mu \mathrm{m}\end{array}$ & $\begin{array}{c}0,007 \\
\text { sur } \\
210 \mu \mathrm{m}\end{array}$ \\
\hline
\end{tabular}

où $\mathrm{d}\left(\frac{\mathrm{n}_{\mathrm{Cr}}}{\mathrm{S}}\right)$ est le nombre de moles de $\mathrm{Cr}$ par unité de surface arrivant sur le front d'oxydation pendant dt en fin de palier pour former $\mathrm{Cr}_{2} \mathrm{O}_{3}$ avec l'oxygène dont la cinétique d'arrrivée est liée à celle de la prise de masse corrigée de la volatilisation, $\mathrm{D}_{\mathrm{Cr}}$ est le coefficient de diffusion de $\mathrm{Cr}$ au travers de la zone décarburée, et $\|\overrightarrow{\operatorname{grad}}[\mathrm{Cr}]\|$ est le module du gradient de la concentration du chrome $[\mathrm{Cr}]$ (cette dernière s'exprimant en moles par unité de volume).

Le second membre de cette équation est égal à $\mathrm{D}_{\mathrm{Cr}} \cdot\left(\frac{\rho_{\mathrm{all}}}{\mathrm{M}_{\mathrm{Cr}}}\right) \cdot\left(\frac{\Delta \%_{\mathrm{m}} \mathrm{Cr}}{\Delta \mathrm{x}}\right)$ c'est-à-dire à $\mathrm{D}_{\mathrm{Cr}}$ multiplié par le produit de d'une part la masse volumique de l'alliage divisée par la masse molaire du chrome, et d'autre part du module du gradient moyen de la teneur massique en chrome.

Le premier membre est égal à la valeur finale (c'est-à-dire à $\mathrm{t}=50$ heures) de la quantité $\frac{2}{3} \cdot \frac{\mathrm{d}\left(\frac{\mathrm{n}_{\mathrm{O}}}{\mathrm{S}}\right)}{\mathrm{dt}}$, notée $\left.\frac{2}{3} \cdot \frac{\mathrm{d}\left(\frac{\mathrm{n}_{\mathrm{O}}}{\mathrm{S}}\right)}{\mathrm{dt}}\right|_{\text {fin }}, \frac{\mathrm{n}_{\mathrm{O}}}{\mathrm{S}}$ étant le nombre par unité de surface de moles d'oxygène impliqué dans la croissance de la chromine, soit à $\left.\frac{2}{3 \mathrm{M}_{\mathrm{O}}} \cdot \frac{\mathrm{d}\left(\frac{\mathrm{m}_{\mathrm{O}}}{\mathrm{S}}\right)}{\mathrm{dt}}\right|_{\text {fin }}$ ou $\left.\frac{2}{3 \mathrm{M}_{\mathrm{O}}} \cdot \sqrt{\frac{\mathrm{Kp}}{2 . \mathrm{t}}}\right|_{\text {fin }}$.

Le coefficient de diffusion du chrome peut alors s'exprimer au moyen de l'équation (14) :

$$
\mathrm{D}_{\mathrm{Cr}}=\frac{2 \cdot \mathrm{M}_{\mathrm{Cr}}}{3 \cdot \mathrm{M}_{\mathrm{O}}} \cdot \frac{\sqrt{\left.\frac{\mathrm{Kp}}{2 \cdot \mathrm{t}}\right|_{\text {fin }}}}{\rho_{\text {all }} \cdot \frac{\Delta \%_{\mathrm{m}} \mathrm{Cr}}{\Delta \mathrm{x}}}
$$

dans laquelle figurent les masses molaires atomiques de l'oxygène et du chrome, la constante parabolique locale $\left(\mathrm{Kp}_{/ /}\right.$ou $\mathrm{Kp} \perp$, tableau III), la masse volumique de l'alliage $\left(8,2 \mathrm{~g} . \mathrm{cm}^{-3}\right)$ et le gradient moyen de teneur massique en chrome au travers de la zone considérée. 
Les valeurs de coefficients $\mathrm{D}_{\mathrm{Cr}}$ ainsi déterminées sont données dans le tableau VII. A l'exception de l'alliage $\mathrm{Ni30Cr} 0,2 \mathrm{C}$ pour lequel une valeur surprenante a été obtenue pour l'orientation parallèle à $1000^{\circ} \mathrm{C}$, le coefficient de diffusion augmente logiquement avec la température, mais surtout il est plus élevé pour l'alliage contenant le plus de carbures (Ni30Cr0,8C), et il est aussi plus élevé pour l'orientation perpendiculaire que pour l'orientation parallèle, à l'exception de l'alliage Ni30Cr0,2C à $1200^{\circ} \mathrm{C}$ (égalité des deux valeurs du coefficient).

Tableau VII. Coefficients de diffusion du chrome déduits des vitesses de prise de masse et des profils de concentration en chrome

\begin{tabular}{|c|c|c|c|c|}
\hline \multirow{2}{*}{$\begin{array}{c}\mathbf{D}_{\mathrm{Cr}} \\
\left(10^{-11} \mathrm{~cm}^{2} / \mathrm{s}\right)\end{array}$} & $1000^{\circ} \mathrm{C}$ & $1100^{\circ} \mathrm{C}$ & $1200^{\circ} \mathrm{C}$ \\
\hline \multirow{2}{*}{$\begin{array}{c}\mathrm{Ni} 30 \mathrm{Cr} \\
0,2 \mathrm{C}\end{array}$} & $\perp$ & 6,6 & 15 & 138 \\
\hline \multirow{2}{*}{$\begin{array}{c}\mathrm{Ni} 30 \mathrm{Cr} \\
0,8 \mathrm{C}\end{array}$} & $\perp$ & 2,9 & 21 & 139 \\
\cline { 2 - 5 } & $\perp$ & 8,1 & 44 & 162 \\
\hline
\end{tabular}

\section{DISCUSSION}

La cinétique d'oxydation des alliages base nickel formeurs de chromine a fait l'objet de très nombreux travaux, depuis plusieurs dizaines d'années jusqu'à très récemment [10-15]. Parmi ceuxci figurent certains portant plus particulièrement sur les alliages binaires $\mathrm{Ni}-\mathrm{xCr}$, qui se rapprochent des deux alliages de la présente étude. Aussi est-il déjà intéressant, à titre préliminaire, de comparer les constantes d'oxydation paraboliques obtenues avec quelques-unes de celles trouvées antérieurement [13]. Le tableau VIII permet de constater la bonne cohérence entre les valeurs mesurées pour une même température, malgré que la pression partielle en dioxygène ait été différente.

Tableau VIII. Valeurs des constantes paraboliques obtenues dans cette étude, en comparaison avec des données antérieures de la littérature

\begin{tabular}{|c|c|c|c|}
\hline $\begin{array}{c}\mathrm{Kp} \\
\left(10^{-12} \mathrm{~g}^{2} \cdot \mathrm{cm}^{-4} \cdot \mathrm{s}^{-1}\right)\end{array}$ & $1000^{\circ} \mathrm{C}$ & $1100^{\circ} \mathrm{C}$ & $1200^{\circ} \mathrm{C}$ \\
\hline $\begin{array}{c}\text { Cette étude } \\
\text { (Ni30Cr0,2C } \\
\text { et Ni30Cr0,8C) }\end{array}$ & 4,9 à 12 & 33 à 69 & 99 à 142 \\
\hline $\begin{array}{c}{[13]} \\
\mathrm{Ni}-30 \mathrm{Cr} * \\
\left(\mathrm{P}_{\mathrm{O} 2}=1 \mathrm{~atm}\right)\end{array}$ & 1,4 & 45 & 110 \\
\hline
\end{tabular}

* : les valeurs numériques données dans [13] sont relatives à l'expression $\mathrm{m} / \mathrm{S}(\mathrm{t})=\sqrt{\mathrm{Kp}} \cdot \sqrt{\mathrm{t}}$ et non à l'expression $\mathrm{m} / \mathrm{S}(\mathrm{t})=\sqrt{2 \cdot \mathrm{Kp}} \cdot \sqrt{\mathrm{t}}$ comme dans la présente étude ; elles ont donc été divisées par 2 
L'orientation dendritique a visiblement un effet sur la cinétique d'oxydation locale des alliages de fonderie classique. Les différences apparaissent dès les courbes thermogravimétriques brutes, mais deviennent plus franches à la vue des constantes linéaires et des constantes paraboliques, surtout à l'issue du découplage des constantes qui a permis de séparer les contributions des deux types de surface. Ce fait a ensuite été confirmé par les observations et mesures réalisées sur la couche de chromine externe. Le fait que l'ordre des cinétiques entre parallèle et perpendiculaire varie avec la température devient explicable à la vue des deux niveaux d'énergie d'activation qui semblent différents (environ $180 \mathrm{~kJ}^{\mathrm{mol}}{ }^{-1}$ pour l'orientation parallèle et $240 \mathrm{~J} . \mathrm{mol}^{-1}$ pour l'orientation perpendiculaire), même si ces valeurs doivent être considérées avec prudence du fait que seules trois températures aient été considérées et que l'alignement des points en représentation d'Arrhénius ait été perfectible dans certains cas.

De telles différences de cinétique ont été antérieurement rencontrées pour des alliages de compositions et de microstructures plus complexes [5], qui avaient conduit à des cinétiques généralement plus faibles que pour les alliages simples étudiés ici. Pour ces superalliages à base de nickel et à base de cobalt l'ordre des deux constantes $\mathrm{Kp}$ correspondant aux deux orientations dendritiques variait suivant la nature et la composition chimique de l'alliage. Par exemple, la constante $\mathrm{Kp}$ à 1000 et $1100^{\circ} \mathrm{C}$ de l'alliage base cobalt renforcé par des carbures de tantale et par des carbures de chrome était plus grande pour l'orientation perpendiculaire que pour l'orientation parallèle, alors que le contraire était observé pour un superalliage base nickel renforcé par des carbures de chrome et de tungstène.

Comme cela avait déjà été constaté dans le cas des alliages précédents, une zone décarburée se développe depuis l'extérieur de l'échantillon pendant son oxydation, ici aussi sur une profondeur tendant à être plus grande pour l'orientation parallèle que pour l'orientation perpendiculaire. Pour l'alliage à $0,2 \% \mathrm{C}$ cette zone décarburée devient plus étendue que pour celui à $0,8 \% \mathrm{C}$, uniquement après 50 heures à $1200^{\circ} \mathrm{C}$, alors que cette différence existait aussi à plus basse température pour les superalliages de compositions plus complexes. Ceci est peut-être dû à la durée d'oxydation qui est plus courte dans le cas présent (50 heures contre 100 pour les superalliages).

Les profils de concentrations réalisés à la microsonde sur l'entière profondeur affectée par l'oxydation (notamment incluant la zone décarburée) ont montré que la zone décarburée est siège d'un fort gradient de concentration en chrome, par comparaison avec la zone voisine existant plus en profondeur et dans laquelle les carbures de chrome existent toujours et demeurent quasiment inchangés. Ceci est lié au fait bien connu que les carbures de chrome interdendritiques favorisent la diffusion du chrome en direction du front d'oxydation, un effet qui conduit aussi à un deuxième gradient (zone ayant conservé ses carbures) plus faible dans le cas de l'alliage Ni30Cr0,8\%C que pour l'alliage Ni30Cr0,2C. Il apparaît aussi que l'orientation perpendiculaire facilite la diffusion du chrome en comparaison avec l'orientation parallèle, et ce pour les deux alliages étudiés. En utilisant les normes moyennes de gradients au travers de la zone décarburée, ainsi que la vitesse d'oxydation à la fin des essais thermogravimétriques, il a été précisément possible d'estimer les coefficients de diffusion moyens du chrome au travers de la zone décarburée. Les résultats ainsi obtenus confirment les déductions précédentes : la diffusion du chrome serait d'autant plus facile quand le réseau de carbures de chrome est plus développé et aussi quand les dendrites sont perpendiculaires à la surface oxydée que quand elles lui sont parallèles.

Le tableau IX permet la comparaison entre les coefficients de diffusion du chrome globaux (au sens qu'ils représentent ensemble la diffusion en volume et la diffusion aux joints de grains sans les dissocier) et des valeurs obtenues antérieurement [12,13] par d'autres méthodes (en l'occurrence la méthode de Gruzin généralisée). On constate que les valeurs obtenues dans cette étude sont du même ordre de grandeur que les résultats antérieurs, mais tout de même supérieurs à ces derniers. Ceci peut venir d'un caractère d'autant plus favorable à la diffusion intergranulaire du chrome dans la zone décarburée des alliages de cette étude que celle-ci contenait initialement plus de carbures et que l'orientation des joints de grains est perpendiculaire à la surface oxydée plutôt que parallèle. En 
effet il est possible qu'un plus grand désordre structural occasionné dans les joints de grains par la dissolution de davantage de carbures permette une diffusion plus facile, surtout si ces zones intergranulaires sont en moyenne bien orientées relativement à la direction générale de diffusion du chrome induite par l'oxydation en surface de l'alliage. Moyennant cette hypothèse, les valeurs de coefficient de diffusion déterminées dans la présente étude seraient a priori cohérentes avec les résultats antérieurs et ceci permettrait d'affirmer qu'en effet la diffusion du chrome en direction du front d'oxydation est facilitée par un réseau de carbures interdendritiques plus dense et par une orientation des dendrites perpendiculaire à la surface extérieure.

On peut aussi se poser la question d'une possible action du nickel sur certains phénomènes intervenant dans le processus général de l'oxydation, qui serait différenciée suivant l'orientation dendritique. En effet cet élément est capable de diffuser très vite et peut intervenir au tout début de l'oxydation, par son oxyde préliminairement formé ou par dopage partiel de la chromine. Comme l'épaisseur d'alliage affecté par l'oxydation est alors encore très faible, l'orientation des dendrites ne peut à ce moment même avoir une influence sensible. De ce fait, l'action du nickel sur les étapes préliminaires comme sur la poursuite du processus d'oxydation ne peut pas dépendre de l'orientation des dendrites.

Tableau IX. Valeurs des coefficients de diffusion du chrome au travers de la zone décarburée obtenus dans cette étude, en comparaison avec des données antérieures de la littérature

\begin{tabular}{|c|c|c|c|c|}
\hline $\begin{array}{c}\mathrm{D}_{\mathrm{Cr}} \\
\left(10^{-11} \mathrm{~cm}^{2} \cdot \mathrm{s}^{-1}\right)\end{array}$ & $900^{\circ} \mathrm{C}$ & $1000^{\circ} \mathrm{C}$ & $1100^{\circ} \mathrm{C}$ & $1200^{\circ} \mathrm{C}$ \\
\hline $\begin{array}{c}\text { Cette étude } \\
(\mathrm{Ni30Cr0}, 2 \mathrm{C} \\
\text { et Ni30Cr0,8C) }\end{array}$ & $/$ & 3 à 8 & 15 à 93 & 138 à 350 \\
\hline$[13]$ & 0,21 & $/$ & $/$ & 44 \\
\hline$[12]^{*}$ & 0,12 & 0,96 & 5,9 & 28 \\
\hline
\end{tabular}

* : valeurs obtenues à l'aide de l'expression $\mathrm{D}_{\mathrm{Cr}}=0,61 . \exp [-\mathrm{Q} /(\mathrm{R} . \mathrm{T})]$ avec $\mathrm{Q}=-63 \mathrm{kcal} / \mathrm{mol}$ donnant le coefficient de diffusion volumique du $\mathrm{Cr}$ dans un alliage binaire $\mathrm{Ni}-20 \mathrm{Cr}$.

Au sujet du grossissement des carbures existants ou de la précipitation de nouveaux carbures dans une zone de faible épaisseur séparant la zone décarburée du reste de l'alliage, il s'agit d'un phénomène déjà observé antérieurement, et expliqué comme suit [16,17]. A certaines températures, tout ou partie du carbone issu des carbures en cours de décomposition du fait de l'oxydation (diffusion de leurs éléments constitutifs $\mathrm{Cr}$, ou autres tels que Ta pour les alliages qui en contiennent), peut migrer en direction de l'intérieur de l'alliage. Si la température est assez basse (vers $1000^{\circ} \mathrm{C}$ ), cette diffusion peut se limiter à une région voisine dans laquelle l'enrichissement local en carbone peut provoquer deux phénomènes différents. Il peut premièrement s'agir d'une modification des carbures interdendritiques pré-existants (croissance des carbures ou changement de leur stoechiométrie pour une plus grande richesse en carbone $\mathrm{Cr}_{23} \mathrm{C}_{6} \rightarrow \mathrm{Cr}_{7} \mathrm{C}_{3}$ ). Deuxièmement ce sont de nouveaux carbures qui peuvent précipiter au cœur de la matrice à partir du chrome présent en solution solide, avec une forme aciculaire. Observée au MEB en mode électrons rétrodiffusés, la zone concernée contient alors des carbures plus grossiers qu'à cœur, plus sombres ou additionnés de nouveaux carbures intragranulaires. Cette fraction supplémentaire de carbures, qu'elle résulte d'un grossissement des carbures existants ou d'une précipitation de nouveaux carbures, est principalement obtenue pendant le palier isotherme lui-même, même si par la suite une nouvelle sursaturation de la matrice en carbone apparaissant au cours du refroidissement puisse 
entraîner une poursuite du phénomène. En effet, pendant le refroidissement à $10^{\circ} \mathrm{C} / \mathrm{min}$, la durée du séjour à température encore suffisamment élevée pour le permettre cinétiquement, est bien plus courte que la durée du palier, alors qu'au cours de ce dernier cinétiques de diffusion comme de précipitation étaient de plus maximales pour permettre à l'alliage d'atteindre dans cette zone enrichie en carbone la microstructure correspondant à la nouvelle composition (nouvelle microstructure conforme aux résultats de calcul de modélisation thermodynamique). Quand la température est suffisamment élevée, on peut penser que soit le carbone a pu diffuser bien plus profondément dans l'alliage sur une étendue plus grande où il n'entraîne pas de surconcentration sensible, soit qu'il a simplement été oxydé et qu'il a quitté l'alliage sous forme gazeuse.

Dans le cas des présents alliages, le carbone semble donc avoir aussi diffusé vers l'intérieur à 1000 et $1100^{\circ} \mathrm{C}$, mais peut-être de manière différente suivant la teneur initiale en carbone dans l'alliage et suivant l'orientation dendritique, à la vue des différences constatées sur le plan des modifications de carbures. En effet ces modifications sont plus visibles dans le cas de l'alliage $\mathrm{Ni30Cr} 0,2 \mathrm{C}$ que dans celui de l'alliage $\mathrm{Ni30Cr} 0,8 \mathrm{C}$, et plutôt pour l'orientation parallèle que pour l'orientation perpendiculaire. Comme pour la diffusion du chrome en direction de l'extérieur, la diffusion du carbone en direction de l'intérieur serait d'autant plus difficile que l'alliage est moins chargé en carbures interdendritiques et que les dendrites sont plutôt parallèles à la surface.

Ainsi, l'orientation des dendrites conditionnerait la facilité de diffusion du chrome en direction de front d'oxydation (et celle du carbone en direction du cœur de l'alliage), ceci avec des conséquences sur les profils de concentration du chrome, sur l'étendue de la zone décarburée, et sur la modification de la nature et/ou de la population des carbures en bordure de cette dernière zone. Il est probable que cette dépendance de la diffusion du chrome et de celle du carbone vis-à-vis de l'orientation dendritique soit à la source des différences constatées entre vitesses d'oxydation, linéaire ou parabolique, ainsi qu'entre énergies d'activation.

Dans le cas de l'alliage faiblement chargé en carbone, la plus grande rapidité de l'oxydation de la surface parallèle aux dendrites en comparaison avec l'orientation perpendiculaire, peut provenir de la plus difficile diffusion du chrome qui peut nuire à la qualité de la couche protectrice formée et donc au caractère protecteur de cette dernière. Par contre, vu la faible teneur en carbone ou la faible densité des carbures intergranulaires dans cet alliage, le rôle du carbone est peut-être négligeable, même si les phénomènes observés au sujet des carbures frontaliers entre zone décarburée et cœur de l'alliage sont de plus grande ampleur dans le cas parallèle que dans le cas perpendiculaire. Le rôle du carbone est par contre sans doute amplifié dans le cas de l'alliage à 0,8\%C. Sa diffusion vraisemblablement plus facile dans le cas perpendiculaire lui permettrait d'une part de moins s'accumuler localement, d'où de plus faibles conséquences locales du point de vue des carbures, mais aussi l'exposerait d'autre part peut-être davantage à l'oxydation. Son dégagement sous forme de gaz, plus intense dans le cas perpendiculaire, pourrait perturber davantage la croissance de la chromine externe dans le cas perpendiculaire que dans le cas parallèle, avec pour conséquence une perméabilité plus grande de cette dernière vis-à-vis de la conduction ionique ou même de la pénétration de l'air lui-même. Il en résulterait alors, pour la surface perpendiculaire à ses dendrites sous-jacentes, une vitesse d'oxydation plus grande que celle d'une surface qui serait parallèle aux dendrites.

Ces explications possibles ne peuvent rester pour le moment qu'hypothétiques mais elles méritent de faire ultérieurement l'objet d'une étude plus approfondie, notamment en analysant les éventuelles différences de qualité et de structure des oxydes extérieurs formés. Il faudrait parvenir à préserver ces derniers en plus grande quantité à la surface des échantillons après essai afin de connaitre sans ambiguïté leur lieu de formation : surface parallèle ou surface perpendiculaire aux dendrites sous-jacentes. 


\section{CONCLUSION}

Pour des alliages simples aussi, l'orientation des dendrites sous-jacentes influence manifestement la cinétique d'oxydation de la surface correspondante. Ce fait, déjà observé pour des superalliages plus complexes, a pu être mis en relation avec l'effet de cette orientation sur la diffusion du chrome, seul élément métallique hautement oxydable dans la composition de ces alliages ternaires base nickel qui sont notamment dépourvus d'éléments du genre tungstène ou tantale. Il est possible aussi que le carbone, dont le comportement diffusionnel semble aussi dépendre de l'orientation dendritique, puisse également intervenir, mais négativement dans son cas, sur la qualité de la couche de chromine formée. Cependant la perte systématique de la couche de chromine extérieure au refroidissement post-essai ne permet pas l'étude d'éventuelles différences de qualité ou de texture entre les oxydes formés sur l'une et sur l'autre des surfaces d'orientation différentes, en vue d'expliquer plus complètement l'effet de l'orientation sur la rapidité d'oxydation.

\section{BIBLIOGRAPHIE}

[1] G. Lesoult, Solidification. Macrostructures et qualité des produits M59. Techniques de l'Ingénieur (1989).

[2] G. Lesoult, Solidification. Cristallisation et microstructures M58. Techniques de l'Ingénieur (1986).

[3] W. Kurz, D.J. Fisher, Fundamentals of solidification. Trans. Tech. Publ. (1989).

[4] P. Kofstad, High temperature corrosion. Elsevier applied science (1988).

[5] P. Sarrazin, A. Galerie, J. Fouletier, Les mécanismes de la corrosion sèche, une approche cinétique, EDPSciences (2000).

[6] P. Berthod, S. Raude, A. Chiaravalle, A.S. Renck, C. Rapin, R. Podor, Influence de la texture microstructurale de superalliages de fonderie sur leur comportement en oxydation à haute température, Rev. Mét. - C.I.T. / Sci. et Génie Mat. 12 (2004) 1031-1042.

[7] E.F. Bradley, Superalloys: A technical guide. ASM International (1988).

[8] C.T. Sims, W.C. Hagel, The superalloys. John Wiley \& Sons (1972).

[9] J. Di Martino, Oxydation à haute température et corrosion par le verre $\mathrm{C}_{3}$ de superalliages base cobalt, Thèse de l'Université Henri Poincaré Nancy I (2002).

[10] C.S. Giggins, F.S. Pettit, Oxidation of Ni-Cr alloys between $800^{\circ} \mathrm{C}$ and $1200^{\circ} \mathrm{C}$, Met Soc of AIME-Trans 245/12 (1969) 2495-2507.

[11] C.S. Giggins, F.S. Pettit,Effect of alloy grain-size and surface deformation on the selective oxidation of chromium in Ni-Cr alloys at temperatures of $900^{\circ} \mathrm{C}$ and $1100^{\circ} \mathrm{C}$, Met Soc of AIME-Trans 245/12 (1969) 2509-2514.

[12] P. Moulin, A.M. Huntz, G. Béranger, P. Lacombe, Rôle des phénomènes diffusionnels en volume et intergranulaire sur l'oxydation entre 800 et $1300^{\circ} \mathrm{C}$ d'un alliage $\mathrm{Ni}-\mathrm{Cr} 80 / 20$, Scripta Metall. 11 (1977) 533-537.

[13] P. Moulin, A.M. Huntz, P. Lacombe, Influence des phénomènes diffusionnels sur le mécanisme d'oxydation des alliages Ni-Cr, Acta Metall. 28 (1980) 745-756. 
[14] V.B. Trindade, U. Krupp, Ph.E.G. Wagenhuber, H.J. Christ, Oxidation mechanisms of Crcontaining steels and Ni-base alloys at high-temperatures - Part I: The different role of alloy grain boundaries, Materials and Corrosion 56/11 (2005) 785-790.

[15] Y.Y. Andreev, A.A. Shumkin, A new theoretical approach to the thermodynamic calculation of high-temperature oxidation of Ni-Cr alloys, Protection of Metals 42/3, (2006) 221-226.

[16] P. Berthod, S. Michon, J. Di Martino, S. Mathieu, S. Noël, R. Podor, C. Rapin, Thermodynamic calculations for studying high temperature oxidation of superalloys, Computer Coupling of Phase Diagrams and Thermochemistry 27 (2003) 279-288.

[17] P. Berthod, C. Vébert, L. Aranda, R. Podor, C. Rapin, Study of carbide transformations during high-temperature oxidation of nickel-base superalloys, Oxidation of Metals, 63 (1/2) (2005) 57-72. 